\title{
Mesoscopic modelling and simulation of espresso coffee extraction
}

\author{
M. Ellero ${ }^{1,2,3, a)}$ and L. Navarini ${ }^{4, b)}$ \\ 1) Basque Center for Applied Mathematics (BCAM), \\ Alameda de Mazarredo 14, 48400 Bilbao, Spain \\ 2) IKERBASQUE, Basque Foundation for Science, \\ Calle de María Díaz de Haro 3, 48013 Bilbao, Spain \\ 3) Zienkiewicz Centre for Computational Engineering (ZCCE), Swansea University, \\ Bay Campus, Swansea SA1 8EN, United Kingdom. \\ 4) Illycaffe' S.p.A, Via Flavia 110, Trieste 34147, Italy
}

A mesoscopic model for the simulation of espresso extraction based on the Smoothed Particle Hydrodynamics method is presented. The model incorporates some essential features such as bimodal granulometry (fines-coarses) of the coffee bed, double (liquid/intra-granular) molecular diffusion and solid-liquid release mechanism. The porous structures ('coarses') are modelled as stationary solid regions whereas the migration of cellular fragments ('fines') is described by single-particles advected by the flow. The boundary filter is modelled as a buffer region where fines are immobilized while entering it, therefore providing a transient flow impedance. The model captures well the transient permeability of the coffee bed under direct-inverse discharge observed in experiments, showing the importance of fines migration on the hydrodynamics of the extraction. The concentration kinetics for different molecular compounds (i.e caffeine, trigonelline and chlorogenic acid) are compared to experimental data for a traditional espresso extraction, showing excellent results. The present work lays down the basis for the virtual analysis of coffee flavors by monitoring the hydrodynamic and microstructural effects on the balance of extracted key-odorant or taste-actives compounds in the beverage.

\footnotetext{
a) Electronic mail: mellero@bcamath.org

b)Electronic mail: luciano.navarini@illy.com
} 


\section{INTRODUCTION}

Coffee is one of the most widely consumed beverages in the world. Several brewing methods can be used to prepare the beverage depending on consumer's taste as well as cultural and geographical habits. In many countries, drip brew, or filter coffee, is the traditionally consumed beverage. This method for brewing coffee involves pouring water over roasted and ground coffee contained on a filter. Water seeps through the coffee, absorbing its extractable fraction solely under gravity, and then passes through the bottom of the filter. The used coffee grounds are retained in the filter with the liquid falling (dripping) into a collecting vessel such as a carafe or pot ${ }^{1}$.

In addition to this popular coffee beverage, espresso coffee is gaining a big world-wide success not only as a phenomenon of fashion but also due to the greater sensory satisfaction it gives to the consumer when compared with coffees prepared with other brewing methods. This success is partly due to the recent spread of portioned systems that make espresso preparation very simple even at home ${ }^{2}$. Traditional espresso brewing requires specialized equipments that have to heat water to a temperature between $92^{\circ} \mathrm{C}$ and $94^{\circ} \mathrm{C}$ and pressurize it to $9 \pm 2$ bar $^{3}$. The process is applied (percolation time) until the beverage volume in the cup meets consumer's personal preference or the regional traditions (or both). For example, in Italy, the volume ranges from $20 \mathrm{~mL}$ or less (ristretto) to $50 \mathrm{~mL}$ or more (lungo), with a typical volume of 20 to $30 \mathrm{~mL}$ for regular espresso shot ${ }^{4}$.

The application of pressure, makes espresso brewing more complex than drip brewing from a physico-chemical process point of view. In particular, during the passage of hot water through the layer of roasted and ground coffee (coffee bed), the following chemical and physical phenomena can be described ${ }^{5},{ }^{6}$ :

- initial imbibition of the porous coffee matrix with consequent irreversible progressive swelling of the coffee particles, this causes a progressive decrease in the porosity of the matrix and therefore an increase in hydraulic resistance. During this process, reversible migration of small coffee particles in the direction of water flow also occurs.

- solubilization of the hydrophilic substances contained in the coffee bed resulting in a progressive increase of density and viscosity of the percolating fluid flow and the concomitant partial erosion of the coffee particles. This includes $\mathrm{CO}_{2}$ (present in the coffee 
bed) solubilization in water at high pressure and temperature leading to supersaturation conditions in the final cup and resulting in the development of bubbles (espresso coffee foam or crema) $)^{7}$.

- stripping of coffee lipids thanks to the pressurized hot water and progressive emulsification of lipophilic substances due to the action of surfactants naturally occurring in roasted coffee, with a further progressive increase in viscosity of the percolating fluid. This whole set of phenomena in addition to lead to polyphasic beverage constituted by a foam layer of small bubbles on top of an emulsion of microscopic oil droplets in an aqueous multicomponent solution with dispersed gas bubbles and solid particles, is extremely complex to be modelled.

Several attempts to model espresso coffee extraction have been reported in the literature. In early studies ${ }^{8,9}$, espresso extraction was described as a two-steps process: (1) transient phase: wetting (water fills in coffee particles voids; inter- and intra-particle gas is removed out of the coffee bed) and percolation (mass transfer between coffee particles and water) occur simultaneously; and (2) extraction steady-state phase resulting from the dynamics of the first one.

Within the transient phase two sub phases can be identified: (i) phase 1a (about 1 second duration) where the flow rate and pressure increase according to Darcy's law; (ii) phase $1 \mathrm{~b}$ (about 4 - 5 seconds duration) where in violation of the Darcy's law - and according to the pump characteristics- pressure increases while flow rate decreases. In this phase coffee bed compacts and coffee fine particles migrates in the flow direction. This sequence occurs until an equilibrium is reached. Pressure and flow rate are thus stabilized. (iii) Steady-state phase 1c (third phase with a duration of 15 seconds onwards) remains until the end of the extraction.

Although modelling and simulation of coffee roasting process have been the subject of several works ${ }^{10-12}$, the complex flow filtration process discussed above, particularly phase 1a, has been scarcely investigated. We mention the early lattice gas computations in $^{8}$ subsequently refined in $^{13}$ - and more recently ${ }^{14}$, where however no transient permeability effects (phase 1b) were reproduced. The models were also used to simulate soluble substances extraction ${ }^{15}$ and chemical description ${ }^{9}$. More recently, attention has been paid in investigating the permeability of roast and ground coffee packed beds in the steady-state 
phase and in modelling the effect of particle size distribution and bed bulk porosity on permeability ${ }^{16}$. This is important since coffee bed permeability is a key parameter affecting extraction due to its relevance in determining the flow rate and hence brewing and residence time.

From a coffee cup quality point of view, however, in addition to model the physics of the espresso extraction, it is necessary to take into the account the modelling of the mass transfer during the process and preferably, the taste-wise chemical compounds mass transfer in order to follow the extraction of solubles (and if possible also of not-solubles) from roasted coffee. Physics of coffee extraction from a flow-through cylinder similar to that found in an espresso machine under water pressure conditions has been modelled in earlier studies by ${ }^{17,18}$ and very recently experimentally validated in $^{19-21}$.

In the latter works an upscaling procedure has been applied to a microscopic balance equations for different phases based on a Representative Elementary Volume (REV) approach. In this approach around every point within the coffee bed, macroscopic properties of the 'homogenized' medium are described by phase averages assuming a scale separation between the smallest pore scale and the size of the RVE. This leads to a continuum description based on a set of partial differential equations for multiple species concentrations which can be solved numerically. In particular, extraction from coffee grains has been modelled via two mechanisms: an initial rapid extraction from damaged cells on the grain surface followed by a slower extraction from intact cells in the grain kernel. The developed model has been parameterized by experimentally measured coffee bed properties enabling to quantitatively reproduce the experimental extraction profile.

It has to be highlighted that the Authors, on the basis of reported similar extraction kinetics for several coffee compounds including trigonelline, caffeine, lipids, among a few others, discussed the possible application of their model to profile the cup quality in terms of brew strength and extraction yield and then considering coffee a "a single component". This approximation, even if representing an excellent starting point to exit the "black box" approach towards the mathematical description of the espresso process, it is still quite far from the desired target to "virtually design and control" the espresso cup quality. It neglects the mesoscopic details (e.g. density, transient morphology) of the coffee bed which is taken into account by phenomenological constitutive laws, e.g. effective permeability parameters. Moreover, the effect of the mesoscopic structures of the packed coffee bed (e.g. complex 
granulometry) on the overall water flow and solute dissolution/transport are averaged-out in continuum-based models. On the other hand, it is becoming progressively clearer that the particle size and distribution significantly affects the extraction kinetics with smaller particles leading to a higher extracted amount of several components, e.g. caffeine and trigonelline, per collected coffee mass ${ }^{22}$ and therefore it is important to have a model that can capture these mesoscopic effects ${ }^{23}$.

The goal of this work is to provide a novel simulation framework to describe coffee espresso extraction taking into account the complex mesoscopic structure of the coffee bed. Complex flow through porous media describing the full microstructure have generally focused on geophysical problems such as in $\mathrm{CO}_{2}$ storage or fossil fuel recovery and have been simulated using a variety of numerical techniques. These range from pore network models, lattice gas and lattice Boltzmann methods, Monte Carlo methods, particle methods (Molecular Dynamics, Dissipative Particle Dynamics, Multi-Particle Collision Dynamics and Smoothed Particle Hydrodynamics), and traditional grid-based computational fluid dynamics coupled with interface tracking ${ }^{24-27}$. For a recent review focused on different particle-based methods for mesoscopic transport processes, including thermal transport, reactive biochemical transport, and ionic transport in mesoscopic systems the reader is referred to ${ }^{28}$.

The numerical techniques adopted in this work to model and simulate the coffee filtration in a full mesoscopic setup is the so-called Smoothed Particle Hydrodynamic (SPH) method. SPH is a popular Lagrangian method to resolve the flow of simple and complex liquids by relying on an kernel-based discretization of prescribed set of partial differential equations (e.g. Navier-Stokes equations for the momentum, advection-diffusion equation for suspended solute etc.) describing the flow locally. This generally leads to a discrete set of ordinary differential equations for fluid particles interacting pair-wisely ${ }^{29,30}$. Due to its Lagrangian meshless character the technique is able to tackle complex geometries, such as those arising in a deformable porous media, as well as to model Lagrangian particulate transport ${ }^{31}$, memory effects in complex fluids ${ }^{32}$ and multiphase flow $^{33}$ in a natural way. The technique has been also generalized to incorporate Brownian fluctuations (when needed) on hydrodynamic variables by using the so-called GENERIC framework ${ }^{34}$. This has allowed it to be extended to the regime of fluctuating hydrodynamics both for simple ${ }^{35,36}$ and complex non-Newtonian fluids $^{37,38}$. GENERIC (an acronym for General Equation for Non-equilibrium Reversible- 
Irreversible Coupling) is a general time-evolution equation for beyond-equilibrium systems which guarantee agreement of its solutions with basic physical laws (i.e. conservations of mass and energy and the approach to equilibrium) ${ }^{39}$.

In the context of flow through porous media, there is a number of applications of SPH mainly for geophysical flow problems relevant to enhanced oil recovery and related problems ${ }^{40-43}$. However, little has been done for complex filtration dynamics in the context of food process modelling. In this work we present a new SPH model of coffee filtration able to describe the entire complex mesoscopic structure of the coffee bed and its potential influence on the flow of the liquid through the filter as well as the transport/release of solute. Complex granulometry of the coffee bed is described on different scales, from the fixed porous medium represented by the large solid grains, to the small free cellular fragments - i.e. the 'fines' down to the molecular solute (e.g. caffeine) modelled via a concentration field. Moreover, for the dynamics of the molecular solute a double porous medium system is considered where the solute's transport/diffusion within liquid and solid phases is taken into account together with a mass transfer model due to stripping from the solid-liquid interface. The mesoscopic model naturally leads to the transient flow permeability effects observed in experiments ${ }^{44}$ as well as its effect on the concentration dynamics - which have been traditionally interpreted in terms of microstructural changes such as pore's swelling or fines particles migration ${ }^{8}$. The structure of the paper is the following one: in Sec. II, the mesoscopic model is presented, in particular the separate models for the liquid phase, solid dispersed phases as well as the interaction with the mechanical filter are discussed. In Sec. III the physical problem is analyzed in detail and the relevant dimensionless variables are discussed: this will guide a proper choice of the SPH model parameters. Sec. IV presents the numerical results for the transient flow permeability as well as the concentration dynamics for different molecular solutes (i.e. characterized by different diffusional behaviours). Validation with experimental data for the cumulative extraction of caffeine, trigonelline and several acids is also performed. Finally, in Sec. V the conclusions are reported together with a perspective view on possible future applications and extensions of the current model. 


\section{MESOSCOPIC PARTICLE MODEL}

In this section the mesoscopic model of coffee filtration is presented. The solid phases will be modelled based on the different characteristic dimensions of the specific components. In particular, in the problem of coffee filtration three main characteristic dimensions are associated to distinct dispersed phases: (i) solid grains $(\approx 450 \mu \mathrm{m})$ representing the fixed porous structure; (ii) the so-called 'fines' $(\approx 30 \mu \mathrm{m})$ representing the flowing cellular fragments and (iii) the molecular components, e.g. volatile or non volatile compounds such as caffeine etc. $(\approx 1-10 \mathrm{~nm})$. Water flowing through the porous structure and coupled with material transport will be modelled based on standard Newtonian hydrodynamics. A sketch of the model is given in Fig. (1).

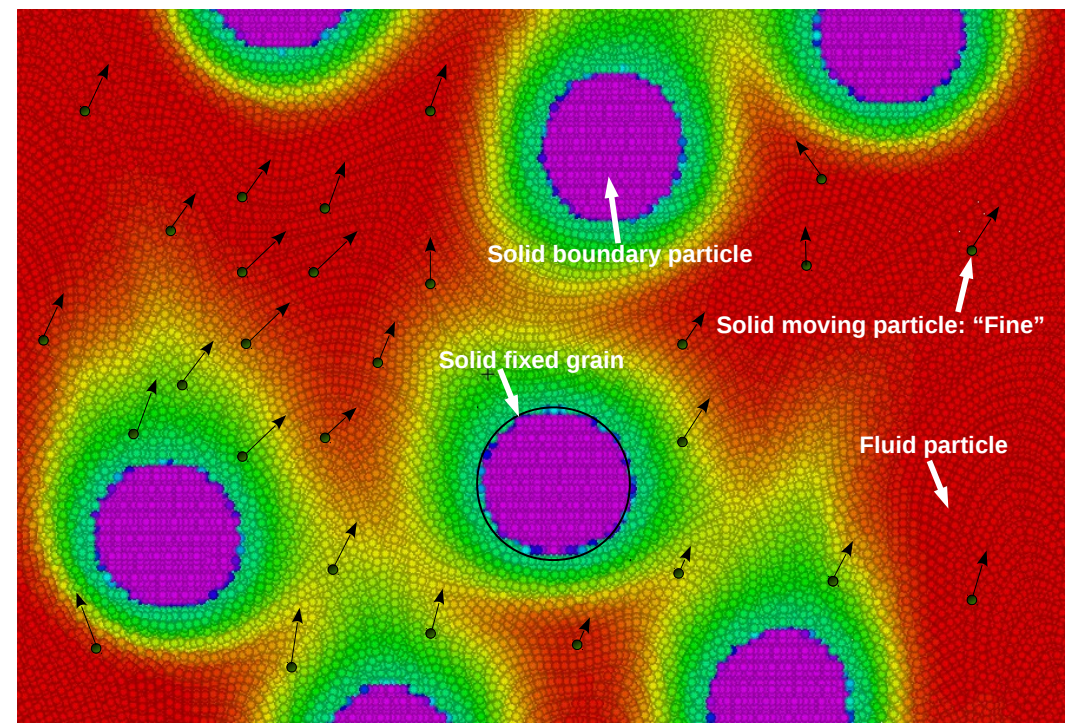

FIG. 1: Sketch of the SPH model: solid dispersed phases is described at different levels. (a) Large solid grains representing the fixed porous structures are denoted as a black circles and are modelled by stationary solid boundary particles located inside the prescribed domain. (b) Small solid fragments, i.e. the "fines", are modelled as independent solid moving particles. (c) The smallest dispersed molecular components - the chemical species - are treated on a continuum level via a concentration field advected by the fluid particles: color map in the figure describes a typical snapshot of the concentration field (violet: maximum - red:zero). Details of the models are given in Sec. II.A-F. 


\section{A. Suspending fluid phase model: Smoothed Particle Hydrodynamics}

The fluid phase dynamics is governed by the isothermal Navier-Stokes equations. The model adopted in this work is the Smoothed Particle Hydrodynamics (SPH) which is a Lagrangian meshless method for the numerical solution of partial differential equations ${ }^{29}$. In SPH a set of fluid particles $i=1, . ., N$ are distributed homogeneously over the domain and move according to conservative and dissipative interparticle forces $\mathbf{F}_{i j}^{C, D}$ estimated from their local neighborhood.

In the isothermal case, the following set of ordinary differential equations for the particle positions, velocities are solved numerically and represent a Lagrangian discretization of the Navier-Stokes equations ${ }^{45}$

$$
\begin{aligned}
& \dot{\mathbf{r}}_{i}=\mathbf{v}_{i}
\end{aligned}
$$

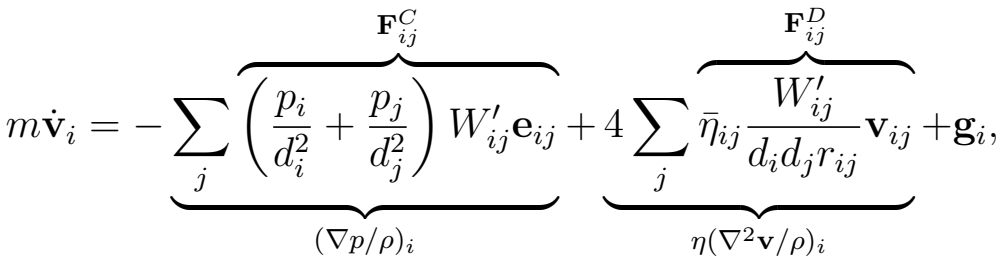

where the time derivative is Lagrangian and $W_{i j}=W\left(r=r_{i j}\right)$ is a kernel function and $W_{i j}^{\prime}=\partial W(r) /\left.\partial r\right|_{r=r_{i j}}$ its spatial derivative. $r_{i j}=\left\|\mathbf{r}_{i j}\right\|=\left\|\mathbf{r}_{i}-\mathbf{r}_{j}\right\|, \mathbf{e}_{i j}=\mathbf{r}_{i j} / r_{i j}$ is the unit vector joining particles $i$ and $j$, whereas $\mathbf{v}_{i j}=\mathbf{v}_{i}-\mathbf{v}_{j}$ their corresponding velocity difference. $\bar{\eta}_{i j}=\left(\eta_{i}+\eta_{j}\right) / 2$ is the averaged dynamic viscosity of the fluid and $\eta_{i}$ is the local value of viscosity associated to particle $i$.

The current formulation allows to model variable local viscosity similar to the model presented recently in Ref. ${ }^{46}$ for shear-thickening fluids. In the simplest case of $\eta_{i}=\eta_{j}=\eta_{0}$ the classical constant viscosity SPH formulation for the Navier-Stokes equations is recovered. $p_{i}$ represents the pressure associated to particle $i$, calculated via a suitable equation of state (EOS). Here we choose an ideal EOS $p_{i}=c_{s}^{2}\left(\rho_{i}-\rho_{0}\right)$ where $\rho_{i}=m d_{i}$ is the mass density associated to the particle $i$ ( $m$ is the constant particle mass) and $c_{s}$ is the sound speed in the liquid. $d_{i}=\sum_{j} W_{i j}$ is the corresponding number density and $\mathbf{g}_{i}$ represents any external body force. Finally, in the previous expression for the EOS the speed of sounds $c_{s}$ must be chosen sufficiently larger than any other velocity present in the problem in order to avoid artificial compressibility effects ${ }^{45}$. 
The SPH model can be generalized to fluctuating hydrodynamics by casting it into the so-called GENERIC formulation ${ }^{34}$ which allows to incorporate additional stochastic terms in Eq.(1) satisfying Fluctuation-Dissipation Theorem ${ }^{35,36}$.

\section{B. Porous solid phase model}

Solid regions of arbitrary shapes can be created by immobilizing a certain number of solid SPH particles located within a prescribed fixed porous structure (i.e. the solid coffee grains) in a similar way to what done in ${ }^{47,48}$ (see Fig.1). No-slip velocity condition is enforced on the liquid-solid interface where boundary particle velocities are set to zero. Solid SPH particles (violet in the figure) interact with fluid SPH particles by means of the same forces presented in Eq.(1), but differently to fluid particles, they are not allowed to move.

This model allows to create porous structures with the prescribed microscopic properties (i.e. size distribution, roughness, solid volume fraction), for example by importing segmented images from experimental data, e.g. obtained via X-ray micro-tomography ${ }^{49,50}$. For sake of simplicity, in this work we consider the internal porous structure of the "toy" coffee bed modelled as a collection of discs arbitrarily distributed over the domain and matching the desired solid volume fraction. It should be remarked that the porous structure could be in principle allowed to move by collecting the total forces and torques exerted on the structure by the surrounding fluid and updating the corresponding coordinate of center of mass as rigid-body translation/rotation in a similar fashion to what presented in Ref. ${ }^{31}$. In this work, a fixed (non-moving) porous solid structure will be considered.

\section{Dispersed molecular phase continuum model}

Due to the large scale separation existing between molecular compounds (e.g. caffeine: $\approx 1 \mathrm{~nm})^{51}$ and the solid grains forming the porous structure $(\approx 450 \mu \mathrm{m})$, the dispersed molecular phase can be treated as a continuum and modelled through a concentration field. In this case, each SPH fluid particle is equipped with an additional microstructural variable, i.e. a scalar concentration field $c_{i}$, whose dynamics is governed by an inhomogeneous advection-diffusion equation. 
We consider here the most general case where the diffusion coefficient $D(\mathbf{r})$ can be spacedependent. The corresponding Lagrangian SPH discretization reads

$$
\dot{c}_{i}=\underbrace{4 \sum_{j} \bar{D}_{i, j} \frac{W_{i j}^{\prime}}{d_{i} d_{j}} \frac{c_{i j}}{r_{i j}}}_{D\left(\nabla^{2} c\right)_{i}}
$$

where the time derivative is Lagrangian and $c_{i j}=c_{i}-c_{j}$ and $\bar{D}_{i, j}=\left(D_{i}+D_{j}\right) / 2$ is the average interparticle diffusion coefficient and $D_{i}$ is the local diffusion coefficient associated to particle $i$. Note that advection is implicitly taken into account through the Lagrangian motion of the particles. Note also that the term within the summation in Eq.(2) is anti-symmetric by swapping $i, j$ indices and therefore the mass of solute is automatically conserved. The same model has been recently successfully applied to the dynamics of cellular components in blood flow ${ }^{52}$.

Fig. (1) shows a color map describing a frame of the concentration field of a given molecular compound released from the solid grains (violet: maximum - red: zero).

Note that different $\bar{D}_{i, j}$ values can be associated to different SPH particles-pairs: this is important, for example, to model the diffusive molecular processes, separately, in the liquid phase and within the solid grain. In fact, Eq.(2) is solved over the entire domain (solid and liquid) with prescribed molecular diffusion coefficients $D_{s}$ (solid phase) and $D_{b}$ (bulk phase). For example, if we assume constant diffusivities within each single phase, we will have $\bar{D}_{i, j}=D_{s}$ for each pair of solid particles $(i, j)$, whereas $\bar{D}_{i, j}=D_{b}$ for each pair of fluid particles. Intra-granular diffusion depends on the specific molecular compound but is typically strongly hindered by the internal cellular structure of the coffee grains ${ }^{53,54}$ and therefore $D_{s}>D_{b}$ is expected ${ }^{19}$.

In practice, several volatile or non-volatile taste-active compounds with different diffusional properties are associated to the final sensorial experience (fruity, malty, honey-like, buttery, roasty etc.). Caffeine, for example, is one of the compounds responsible for the bitterness together with trigonelline, the second most abundant alkaloid in coffee ${ }^{55}$. On the contrary, chlorogenic acids are a class of esters widely distributed in plants and particularly abundant in coffee beans (being mono-caffeoylquinic acid isomers the most important) which are relevant for acidity ${ }^{56}$ and bitterness suppression properties ${ }^{57}$. In the process of coffee filtration it is therefore important to assess the instantaneous time-dependent concentration of different compounds (i.e. characterized by different $D_{b, s}$ ) in the cup, in order to optimize 
the product and/or target specific flavors ${ }^{58}$.

It remains to define the interaction between a pair of solid/liquid particles which defines the molecular transport at the solid-liquid interface. This transport case is known to be not diffusive/osmotic, instead, molecular compounds within the solid at the surface are released in the liquid by a comparably faster uni-directional 'washing' mechanism ${ }^{15}$. To mimic this molecular release mechanism at the solid-liquid interface, we introduce a new release coefficient $D_{r}$ such that $\bar{D}_{i, j}=D_{r}$ only if particle $i$ and $j$ belong to different phases. Moreover, the dynamics governed by the diffusion equation Eq.(2) can allow in principle the transfer of mass from the liquid back to the solid under specific situations such for example a local fluid concentration larger than the solid one (e.g. by accumulation of material downstream). To avoid this back-transport mechanism and to model uniquely the washing release process, we set $\bar{D}_{i, j}=0$ if $c_{i}>c_{j}$ with $i$ being a fluid particle and $j$ being a solid one.

\section{Discrete "fines" model}

"Fines" are modelled as single solid SPH particles advected by the flow. We adopt here the so-called minimal single-particle model proposed in the context of Dissipative Particle Dynamics $^{59,60}$. We select randomly a number of SPH particles in the fluid domain and, according to the target fines concentration, we regard them as a solid flowing particles (black spheres in Fig. (1)). It should be noted that in the bulk flow the fines are just passive tracers and do not have any influence on the flow. However, if their positions are constrained they are characterized by a well-defined hydrodynamic radius (approximately equal to the kernel cutoff radius $r_{c}$ ) and therefore they provide a mechanism of mechanical impedance for the bulk flow itself. This feature is important in the model of the physical filter in the next section.

\section{E. Filter model}

The filter at the bottom (or upper) boundary of the coffee bed is modelled as an additional buffer region of finite thickness (red area in Fig. 2). The fines move with the flow under 


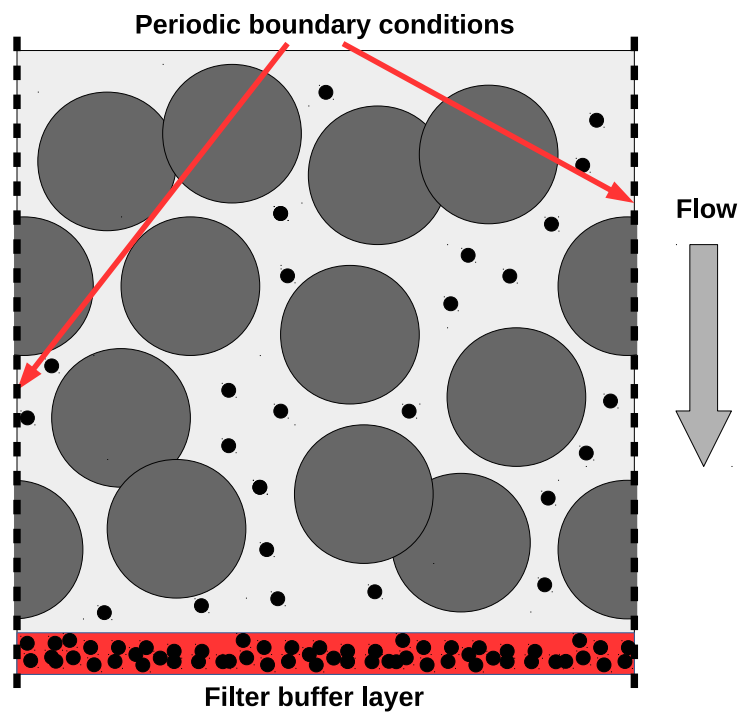

FIG. 2: Sketch of the filter model: fines are advected by the flow. Filter is modelled as a buffer region (red area in the figure) of specified thickness. When fines enter this region they are 'immobilized' and provide transient mechanical impedance to the down-streaming flow depending on the instantaneous concentration of trapped fines.

the action of an external pressure force and eventually percolate the porous structure; when they finally enter the bottom buffer region, their identity change and they are regarded as fixed solid boundary particles with zero velocity - of the same type of those used to model the solid region inside a grain (see Fig. 1). As a consequence, they present an obstacle to the flow of the down-streaming fluid particles which exit the domain. Depending on the initial concentration of fines dispersed in the liquid domain (denoted as $\theta$ ) the total number of fines trapped in the filter can significantly change together with the values of filter resistance leading to a simple model of transient coffee bed permeability ${ }^{44}$. In this filter model, we use $\theta$ as a fitting parameter to match the additional resistance provided by the mechanical filter to the coffee bed, compared to experimental data (see Section IV).

\section{F. Summary}

Summarizing, the present mesoscopic method models different possible physical processes taking place during coffee filtration: (1) hydrodynamic flow through a 2D porous structure; (2) release of a molecular phase (e.g. odorant such as vanillin, linalool etc. or taste-active 
TABLE I: List of model parameters

\begin{tabular}{|c|l|}
\hline \hline Model parameter & Notation \\
\hline fluid mass density & $\rho_{0}$ \\
dynamic fluid viscosity & $\eta$ \\
kinematic fluid viscosity & $\nu$ \\
fluid sound speed & $\mathrm{c}_{s}$ \\
vertical length coffed bed & $\mathrm{H}$ \\
solid grain size & $\mathrm{d}_{\text {grain }}$ \\
solid fine size & $\mathrm{d}_{f i n e}$ \\
grains concentration & $\phi$ \\
fines concentration & $\theta$ \\
molecular diffusivity (fluid) & $\mathrm{D}_{b}$ \\
molecular diffusivity (solid) & $\mathrm{D}_{s}$ \\
molecular washing release (solid/liquid) & $\mathrm{D}_{r}$ \\
pressure body force & $\mathrm{F}_{0}$ \\
average flow velocity & $\mathrm{V}$ \\
\hline \hline
\end{tabular}

compounds such as caffeine, trigonelline and chlorogenic acids etc.) into the flowing liquid phase; (3) diffusion of the target molecular compounds within the liquid/solid phase; (4) migration of solid fines and trapping in the filter: transient permeability. The relevant model parameters are summarized in Table I. Note that dimensionless 'model' parameters are chosen to deliver the correct 'physical' dimensionless variables (e.g. Reynolds, Schmidt, Mach numbers etc.) which allow us to match experimental conditions (see Sec. III.A.).

\section{DIMENSIONAL ANALYSIS OF THE FILTRATION PROCESS}

\section{A. Physical parameters}

In order to compare experimental and numerical results it is necessary to perform a dimensional analysis of the filtration problem. This will guide the choice of parameters of the SPH model shown in Table I. 
Regarding the geometry of the coffee packed bed, we refer to standard conditions described, for example, in Ref. ${ }^{16}$, which approximately corresponds to a cylinder with height $H=1.85 \times 10^{-2} \mathrm{~m}$ and radius $R=1.8 \times 10^{-2} \mathrm{~m}$.

Regarding the granulometric properties of the coffee bed, in most cases an approximate bimodal distribution of the solid phase (excluded the molecular component) is observed with two dominant peaks corresponding to fine particles (fines) and coarser particles (grains), the latter ones representing the fixed porous matrix ${ }^{16,22}$. In Ref. ${ }^{16}$ the two sizes (diameters) are reported to be $d_{\text {fine }} \approx 40-50 \mu \mathrm{m}$ and $d_{\text {grain }} \approx 350-400 \mu \mathrm{m}$. There is however significant variability, depending on the grinding process used to produce the final coffee bed, with values reported in the literature down to $d_{\text {fine }} \approx 30 \mu \mathrm{m}$ and up to $d_{\text {grain }} \approx 450 \mu \mathrm{m}$ (Cappuccio, R.; Suggi Liverani, F. Illycaffeè S.p.A.: internal report). This gives two specific dimensionless length ratios to be matched in simulations, i.e.: $H / d_{\text {grain }} \approx 40-50$ and $d_{\text {grain }} / d_{\text {fine }} \approx 10-15$. Regarding porosity of the coffee bed we consider a conservative choice of solid volume fraction $\phi=0.48$ based on the fixed coarse particles.

Regarding the flow rate measured in Ref. ${ }^{16}$, it is reported to be $\dot{Q} \approx 0.5-3 \times 10^{-5} \mathrm{~m}^{3} \mathrm{~s}^{-1}$ (maximum-peak value; minimum steady value), which is in substantial agreement with the $2-18 \mathrm{~mL} / \mathrm{s}$ reported in Ref. ${ }^{8}$. We consider therefore a low steady flow rate $\dot{Q}_{\min } \approx$ $0.5 \times 10^{-5} \mathrm{~m}^{3} \mathrm{~s}^{-1}$ and a peak flow rate $\dot{Q}_{\max } \approx 3 \times 10^{-5} \mathrm{~m}^{3} \mathrm{~s}^{-1}$, leading to an average steady and peak velocity inside the packed bed $V_{\min }=\dot{Q}_{\min } /\left(\pi R^{2}\right) \approx 0.5 \times 10^{-2} \mathrm{~m} / \mathrm{s}$ and $V_{\max } \approx 3 \times 10^{-2} \mathrm{~m} / \mathrm{s}$.

Now, if we define a microscopic Reynolds number as $\operatorname{Re}=d_{\text {grain }} V / \nu\left(\right.$ where $\nu=10^{-6} \mathrm{~m}^{2} \mathrm{~s}^{-1}$ is the kinematic water viscosity at $25^{\circ} \mathrm{C}$ ), we obtain a minimum-steady and maximum peak Reynolds numbers in the range $\operatorname{Re} \approx 2-12$. Note that this condition corresponds to a fully laminar regime, although not in the Stokes inertial-less limit. Note that during real espresso extraction, temperature distribution can be highly inhomogenous within the coffee bed and can reach values as high as $80^{\circ} \mathrm{C}$. Under these conditions the kinematic water viscosity can be a factor 2 smaller with respect to the value chosen here. This would lead to a maximum operational Reynolds around 20, therefore still in a laminar regime. In Ref. ${ }^{8}$, however, cold water was used for the experiment, in line with the regime considered here.

As discussed above, the solid phase distribution is approximately bimodal with average radius of the fine particle component in the order of $r_{\text {fine }} \approx 15 \mu \mathrm{m}$. These are non-colloidal particles for which the Peclet number is virtually infinite, so Brownian motion does not need 
to be modelled and they can be simply passively tracked along the liquid streamlines. As a matter of fact, if we use the Stokes-Einstein relation to estimate the diffusion coefficient of such a fine particle, we have $D_{\text {fine }}=k_{b} T /\left(6 \pi \eta r_{\text {fine }}\right) \approx 1.46 \times 10^{-14} \mathrm{~m}^{2} \mathrm{~s}^{-1}\left(\eta=10^{-3} \mathrm{~Pa} \cdot \mathrm{s}\right.$ is the water dynamic viscosity) and therefore the microscopic Peclet number for the fines in this filtration process is $\mathrm{Pe}=d_{\text {grain }} V / D_{\text {fine }} \geq 1.3 \times 10^{8}$.

On the other hand, molecular compounds (e.g. caffeine) released from the coffee grains into the flushing solvent are much smaller in size, with resulting bulk diffusion coefficient in water estimated to be $D_{b}=2 \times 10^{-9} \mathrm{~m}^{2} \mathrm{~s}^{-1}$ (depending on the specific compound, concentration and temperature $)^{53,54,61}$. This would lead to a local maximum Peclet number for the advection-diffusion dynamics of caffeine in this filtration process in the order of $\mathrm{Pe}=d_{\text {grain }} V / D_{b} \approx 1000$, which is large but finite. Regions of reduced-flow/stagnation or larger temperature can have local Peclet numbers significantly smaller.

Note also that different substances can have different diffusion coefficients $D_{b}$ in water, in such a way that a differentiated analysis of coffee filtration performance needs to be done based on the effective Peclet number for each substance. For example, some tasteactive and key-odorant compounds can have diffusivities in water substantially larger (e.g. trichloroethylene ${ }^{62}$ ) leading to $\mathrm{Pe} \approx 200$ or smaller under standard espresso extraction conditions. Virtual numerical analysis of compounds release and transport, however, is not restricted to these values and can be in principle explored down to $\mathrm{Pe} \approx 1$, corresponding to extremely slow filtration processes. Note also that all these molecular components have size in the order of few nanometers or less, i.e. much smaller than the fines and coarse particles and therefore they can be regarded as a continuum phase.

Another relevant process affecting the concentration of the solute (e.g. caffeine), would be the rate of release from the solid phase (coffee grains) into the liquid. This is a complex physico-chemical process involving inhomogeneous dispersion of molecular compounds inside a heterogeneous porous solid matrix and its modelling goes well beyond the scope of the current work. However, it is generally considered to be the limiting step in caffeine extraction ${ }^{54}$, being the rate at which it is released into the liquid phase $D_{r}=2 \times 10^{-10} \mathrm{~m}^{2} \mathrm{~s}^{-1}$, i.e. 10 times smaller than free-bulk diffusion ${ }^{54}$. As a consequence of this discussion, the following effective Peclet numbers simulated for this process will be: $\mathrm{Pe}_{b u l k}=d_{\text {grain }} V / D_{b}=\mathrm{Re} \cdot \mathrm{Sc}$ where the Schmidt number is defined as $\mathrm{Sc}=\nu / D_{b} \approx \frac{10^{-6}}{2 \times 10^{-9}} \approx 500$. Therefore Pe $\mathrm{e}_{\text {bulk }} \approx 500-5000$. 
The release mechanism defines another effective $\mathrm{Pe}_{\text {release }} \approx 5000-50,000$, i.e. when $D_{r} \approx 0.1 D_{b}$, as discussed above. The effect of all these parameters on the concentration dynamics will be presented in Sec. IV.

\section{B. Numerical parameters}

In order to model numerically the physical system discussed above, we chose the following setup.

- Coarse coffee grains ('coarses': $500 \mu \mathrm{m}$ ) : they are modelled as fixed spherical solid regions of size $d_{\text {grain }}=2.0$ using $16 \mathrm{SPH}$ particles per diameter (see Fig. 1). The 'coarses' solid volume fraction is $\phi=0.48$.

- Fine particles ('fines': $30 \mu \mathrm{m}$ ): they are modelled as mobile single SPH particles (passive tracers). Being the SPH resolution of the grain 16, we have a computational ratio $d_{\text {grain }} / d_{\text {fine }} \approx 16$ which is consistent with the physical ratio discussed above. The 'fines' solid volume fraction will range in $\theta=0.001-0.01$.

- Molecular compounds (e.g. caffeine, hyperfines etc.: $\ll 1 \mu \mathrm{m})$. Their small size compared to the other characteristic lengths $\left(H, d_{\text {grain }}, d_{\text {fine }}\right)$ justifies a continuum approach based on the solution of an advection-diffusion equation for the corresponding concentration field (Eq.(2)). Different species can in principle have different size and therefore different bulk diffusion coefficients $D_{b}$ as well as solid diffusion coefficients $D_{s}$ and release rates $D_{r}$.

- Coffee bed (2cm thickness):to model a realistic coffee bed of, say, $2 \mathrm{~cm}$ thickness ${ }^{16}$ we consider a height of the simulation domain $L_{y}=80$ ( $y$ is the direction of filtration), leading to a numerical ratio $L_{y} / d_{\text {grain }}=40$, in agreement with that discussed in the previous section. In the transversal direction $(x)$ it is assumed that the coffee bed is homogeneous and periodic boundary conditions can be imposed. This allows to minimize the size of the simulation domain and computing time. Finite size effects can be eliminated by taking $L_{x}=10=5 d_{\text {fine }}$. In conclusion, we consider a two-dimensional domain $L_{x} \times L_{y}=10 \times 80$, discretized with $N=80 \times 640=51,200$ SPH particles.

Fluid density is chosen to be $\rho=1$, whereas viscosity $\mu=3$. The average flow velocity (which controls the effective Reynolds number) is tuned by applying an effective body ac- 
celeration mimicking a pressure drop, i.e. $F=\Delta p /\left(L_{y} \rho\right)$. For example, a value of $F=2000$ leads to peak average velocity $V_{\max } \approx 15$ and peak Reynolds number (in absence of fines: just fixed solid grains) $\operatorname{Re}_{\max }=d_{\text {grain }} V_{\text {max }} \rho / \eta \approx 10$, matching experimental conditions.

The speed of sound is chosen sufficiently large than $V_{\max }$ to reduce density fluctuations, i.e. $c_{s}=500 \gg V_{\max }$.

Finally, the bulk diffusion coefficient for a specific compound will range in $D_{b}=0.005-0.1$ to give a bulk Peclet number $\mathrm{Pe}=600-6000$ corresponding to transient peak flow velocity. Values for the corresponding rate of of release coefficients will be typically a factor 10 smaller than the the bulk free diffusion, i.e. $D_{r}=0.001-0.1$, but different conditions will be explored.

\section{NUMERICAL RESULTS}

\section{A. Inverse filtration process: transient permeability}

In order to understand the different transport processes involved in the percolation of water through the coffee bed system, a transient direct/inverse filtration is considered and results are discussed in relation to experimental data ${ }^{15}$. In this section we focus on the hydrodynamic response of the system. In particular, Fig.3 shows the dynamics of the full
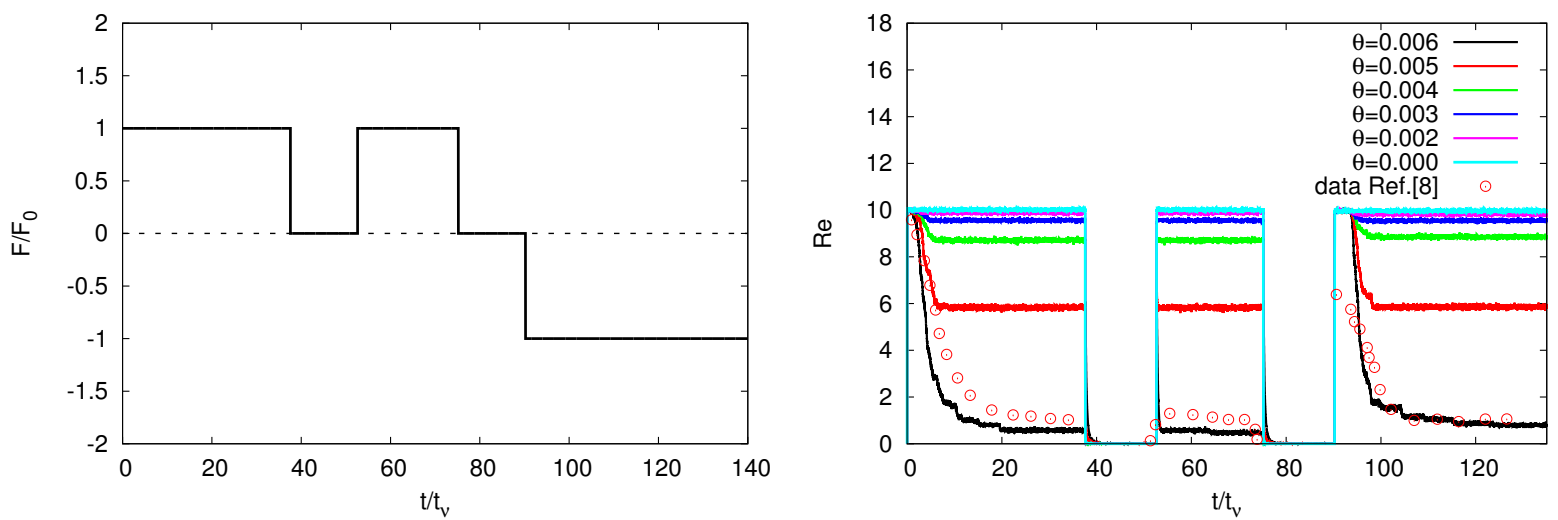

FIG. 3: Inverse discharge. Left: time-dependent dimensionless applied forcing. Right: time-dependent Reynolds number (absolute value) for different fines concentration $\theta$. Slow permeability decay is evident as result of increase fine concentration.

direct/inverse discharge. Left: the time-dependent dimensionless forcing (i.e. pressure drop). 
Right: time-dependent Reynolds number based on spatially-averaged flow velocity (absolute value) for different fines-concentration $\theta$. Experimental data for the transient direct/inverse discharge flow reported in ${ }^{8}$ have been also showed as reference. Note that in the experimental data the initial transient flow increase have been removed since (in experiment) it is due to an applied pressure raising over a finite time, i.e. not 'instantaneously' applied as in the simulations (see Fig. 3: left). Time has been made dimensionless with the viscous liquid time $\tau_{\nu}=d_{\text {grain }}^{2} / \nu=2^{2} / 3=1.33$. In order to compare with experiments, corresponding real viscous time in SI units should be $\tau_{\nu}=d_{\text {grain }}^{2} / \nu=\left(400 \times 10^{-6}\right)^{2} / 10^{-6}=0.16 \mathrm{~s}$.

Initially a direct discharge process with constant pressure forcing is applied up to dimensionless times approximately equal to $t^{*}=t / \tau_{\nu}=38$. This is followed by a resting state (zero applied force for $\left.t^{*}=38-55\right)$ after which the same constant forcing is applied again. Finally, an additional resting condition $\left(t^{*}=75-90\right)$ is followed by a constant pressure force applied in the opposite direction (inverse discharge).

We consider first the flow response of the pure porous structure, i.e. for fine concentration $\theta=0$ (light blue line in Fig.3). After application of pressure drop, flow rate increases quickly and reaches a steady state value $(\operatorname{Re} \approx 10)$. The small transient at $\theta=0$ (not visible in the figure) occurs on typical viscous time scales of order $\tau_{\nu}$. After removal of the forcing, flow rates decay quickly to zero (relaxation time scales $\approx \tau_{\nu}$ ) and then again reach the same value upon re-activation of the forcing in the same direction. After forcing reversal, again the flow responds very quickly reaching the same value of the Reynolds number (absolute value is shown in the figure). Under these conditions $(\theta=0)$, coffee bed permeability is constant and the results are in agreement with previous simulations ${ }^{13}$.

The case where fines are present $(\theta \neq 0)$ is, however, qualitatively different. We have studied different fine concentrations ranging from 0.002 to 0.006 as shown in Fig.3 (right). Initially (direct discharge at $t^{*}<3$ ) the flow reaches the same peak Reynolds number during the very short viscous time scales. This again corresponds to the fast viscous relaxation consistent with a fixed porous structure. In this case, however, it does not represent a steady state but a transient peak. In fact, the meta-stable state at $R e=10$ is followed by a transient decay characterized by a significant longer relaxation time compared to $\tau_{\nu}$. This slow decay $\left(3<t^{*}<10\right)$ is due to the fines be displaced and their transient accumulation in the filter. In fact, fines need some finite time to migrate from their initial positions (randomly dispersed in the liquid phase) to the filter at the boundary of the domain. As 
the number of trapped particles increases, so does the overall flow resistance leading to the observed transient permeability.

Flow rate eventually reaches a steady-state value which depends on the initial concentration of fines present in the liquid phase. Small values of $\theta=0.001$ (violet line) do not alter significant the filtration hydrodynamics respect to simple fixed porous case. However, $\theta=$ 0.006 (black line) leads to a significant reduction of flow permeability (nearly one order in the averaged steady Reynolds number) in substantial agreement with experimental data of espresso extraction reported in Ref. ${ }^{15}$. At longer time (still keeping the forcing term constant) no flow alteration is observed indicating that all fines are migrated to the filter, saturation conditions are reached and no further reduction of resistance is possible.

If the forcing term is temporarily switched off and then re-activated, the flow rate reaches instantaneously the same steady state. This means that the water flows through the same geometrical configuration corresponding to the fixed porous structure with an additional mechanical impedance offered by the unchanged amount of fines trapped in the filter.

Finally, if we invert the flow we observe again the same relaxation process characterized by the long relaxation time as at the beginning of the simulation. The reversed flow forces the fines to move in the opposite direction and be released by the filter, i.e. again through the coffee bed. A typical dimensionless time $\tau_{m}^{*} \approx H /\left(V_{\max } \tau_{\nu}\right)=7.1$ is required for all fines to migrate towards the bottom filter, after which the permeability reaches again a steady state. This is in remarkable good agreement with the transient decay observed in Fig.3 (right) and in experiment of inverse coffee discharge. These results show that fines migration is the main mesoscopic transport process responsible for the reversible transient permeability observed in experiments ${ }^{15,44}$. In fact, although another mechanism could lead to similar increase resistance effect under direct discharge, e.g. the swelling of porous phase, being irreversible it cannot explain the transient peak flow velocity re-obtained under flow reversal ${ }^{15}$.

The corresponding velocity fields inside the porous structure for different $\theta$ are shown in Fig.4. Initial positions for the coarse solid particles have been chosen randomly. Note that different porous structures might lead to slightly different permeabilities (even when the same fixed porosity is considered). Here we specifically tune the applied force $F_{0}$ to obtain a set of dimensionless numbers (Re,Sc etc.) to match the range of experimental values for this specific porous configuration. . 


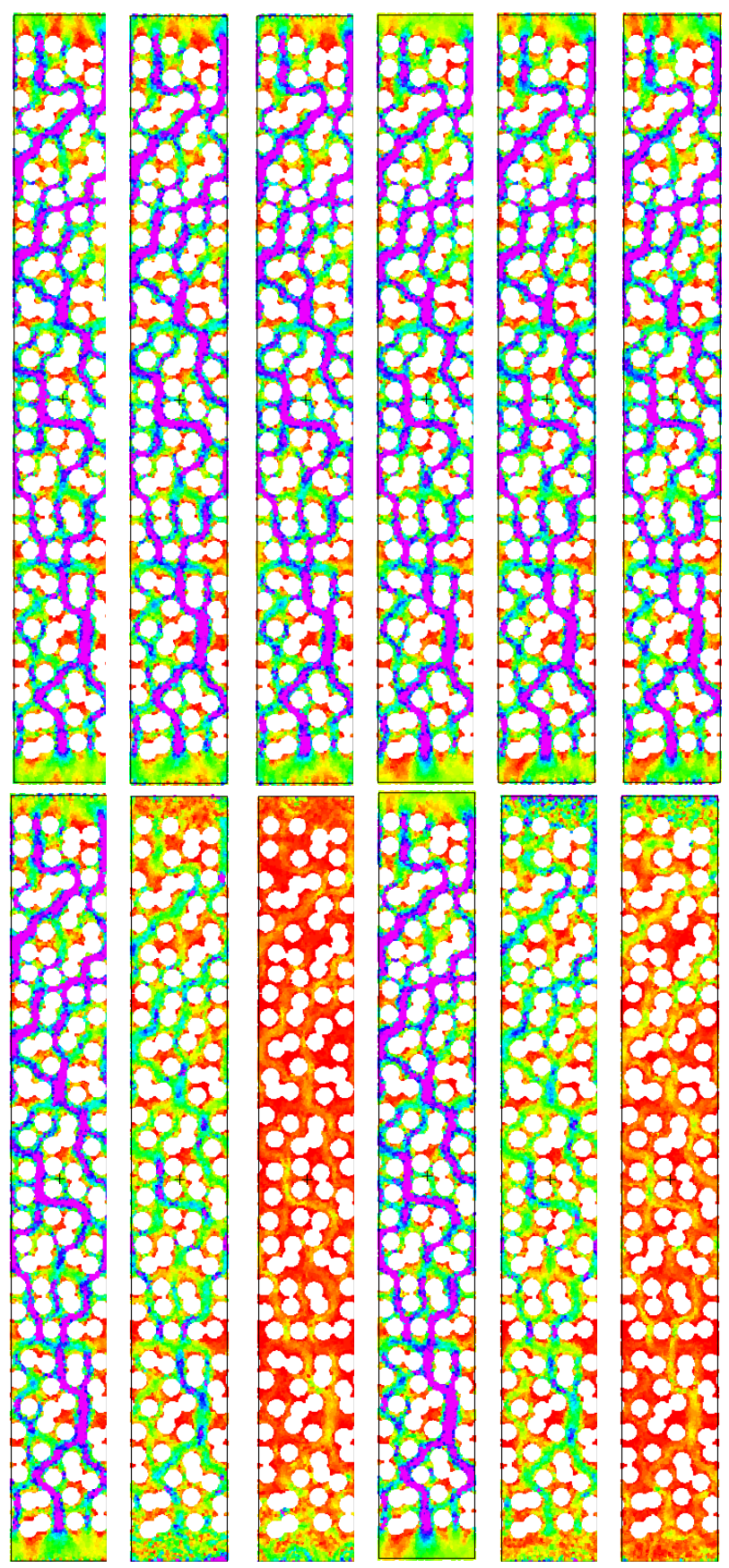

FIG. 4: Velocity field. Top: $\theta=0.002$. Bottom: $\theta=0.006$. Snapshots taken respectively at $t^{*}=1.8,4.8,18$ (direct discharge: left) and $t^{*}=91.8,94.8,108$ (inverse discharge: right).

Top row corresponds to different snapshots for the system with $\theta=0.002$ (violet line in

Fig.3); bottom row corresponds to different snapshots for the system with $\theta=0.006$ (black line in Fig.3). First three frame (from left to right) correspond to dimensionless times $t^{*}=1.8,4.8,18$ (direct discharge) whereas last three frames correspond $t^{*}=91.8,94.8,108$ 


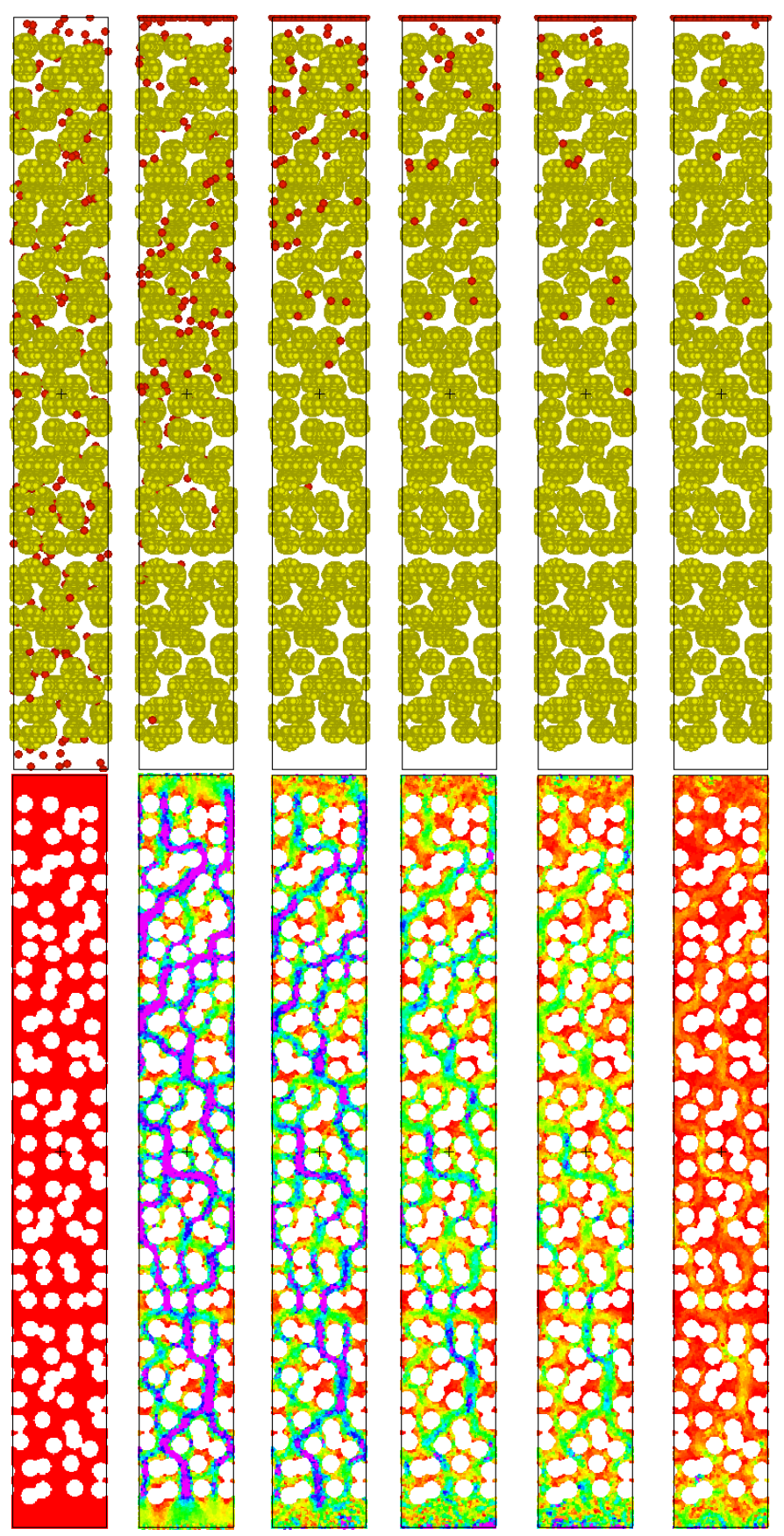

FIG. 5: Direct discharge. $\theta=0.006$. Top: fines distributions. Bottom velocity field. Snapshots taken at $t^{*}=0,1.8,3.6,4.8,6,18$.

(inverse discharge), shown as reference in Fig.3. Regions of local high flow velocity (violet) are visible within the domain. For $\theta=0.002$ a flow through a porous media is reached quickly and remains steady during all the direct discharge stage. For the case $\theta=0.006$ the initial flow field is the same as for $\theta=0.002$, but at larger times it slows down significantly 


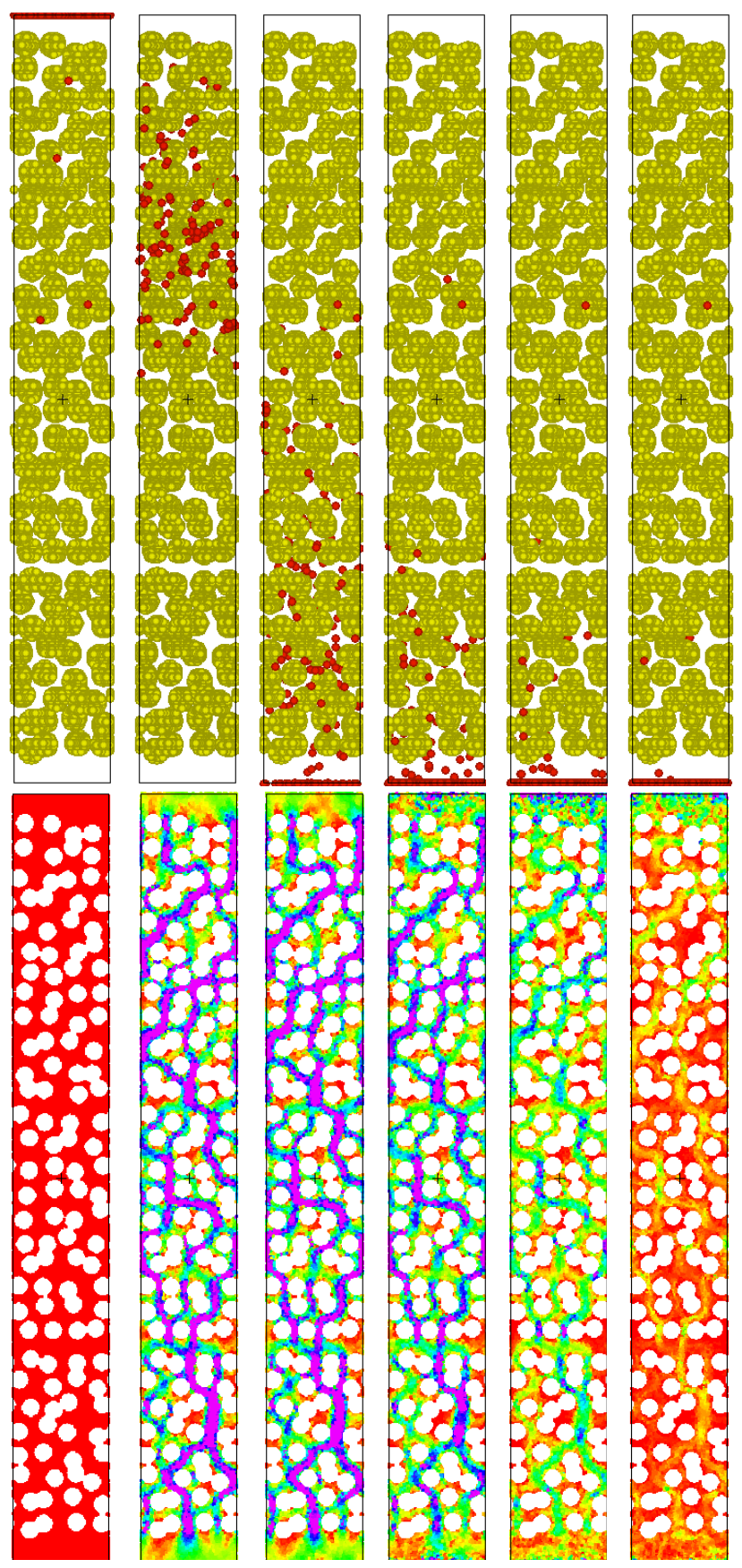

FIG. 6: Inverse discharge. $\theta=0.006$ Top: fines distributions. Bottom: velocity field. Snapshots taken at $t^{*}=90,91.8,93.6,94.8,96,108$.

510 (red areas) keeping the same flow topology. In addition, when the forcing changes direction, 511 unlike the case $\theta=0.002$, for $\theta=0.006$ the flow rate jumps quickly to the peak value ${ }_{512}(\mathrm{Re}=10)$ before reducing again to lower values when the inverse fines migration takes place 513 and they finally reach the opposite filter. 
In order to better clarify the link between the fines microstructure and flow field evolution, in Fig.5 a comparative visual analysis of flow field vs fine microstructure $(\theta=0.006)$ is performed during initial 'direct discharge' (upward fines migration). In Fig.6 the same analysis is done for the final 'inverse discharge' (downward fines migration). Snapshots are taken at times $t^{*}=0,1.8,3.6,4.8,6,18$ (direct discharge) and $t^{*}=90,91.8,93.6,94.8,96,108$ (inverse discharge). From Fig.5 it is evident that as long as the majority of the fines are still migrating, the velocity field reaches the same peak value as for the single porous structure $(\theta=0)$. Only when fines start to accumulate on the upper filter, the magnitude of the velocity field gradually decreases until all fines are trapped in the filter. Upon flow reversal (Fig.6) a counter-migration takes place. The initial peak value of the velocity is reached on the fast time scales $\tau_{\nu}$. The velocity field remains approximately constant until the first fines reach the bottom filter and then - as in the previous case- it starts to decrease in magnitude. This fine-migration mechanism is able to explain quantitatively the transient permeability observed in early experiments with inverse coffee filtration where large transient times were reported in the order of seconds ${ }^{15}$.

\section{B. Concentration dynamics}

After validation of the hydrodynamic response and transient permeability made in the previous section, we study here the release and dynamics of a passive scalar field (the molecular compound concentration, e.g. caffeine) during the filtration process. In particular, we are interested in the resulting cumulative output content in the cup for different choice of physical parameters, namely the concentration of fines $\theta$, the bulk diffusion coefficients $D_{b}$ of the molecular compound, release rates $D_{r}$ and the intra-granular diffusion coefficients $D_{s}$.

The cumulative output percent content is defined as compound-to-total mass ratio, i.e. $M_{\text {compound }}(t) / M_{\text {tot }}(t)$, where both quantities refer to the instantaneous values collected at the output and depend on time. In particular, $M_{\text {tot }}(t)=\int_{0}^{t} \rho_{0} \dot{Q}(t) d t$ where $\dot{Q}(t)=V(t) A$ is the time-dependent flow rate. We consider here the case of direct discharge (Fig.7: left). As discussed previously, to map the present results on real SI units the dimensionless time should be rescaled by the estimated $\tau_{\nu}=0.16 \mathrm{~s}$ based on the main granulometric mode. 

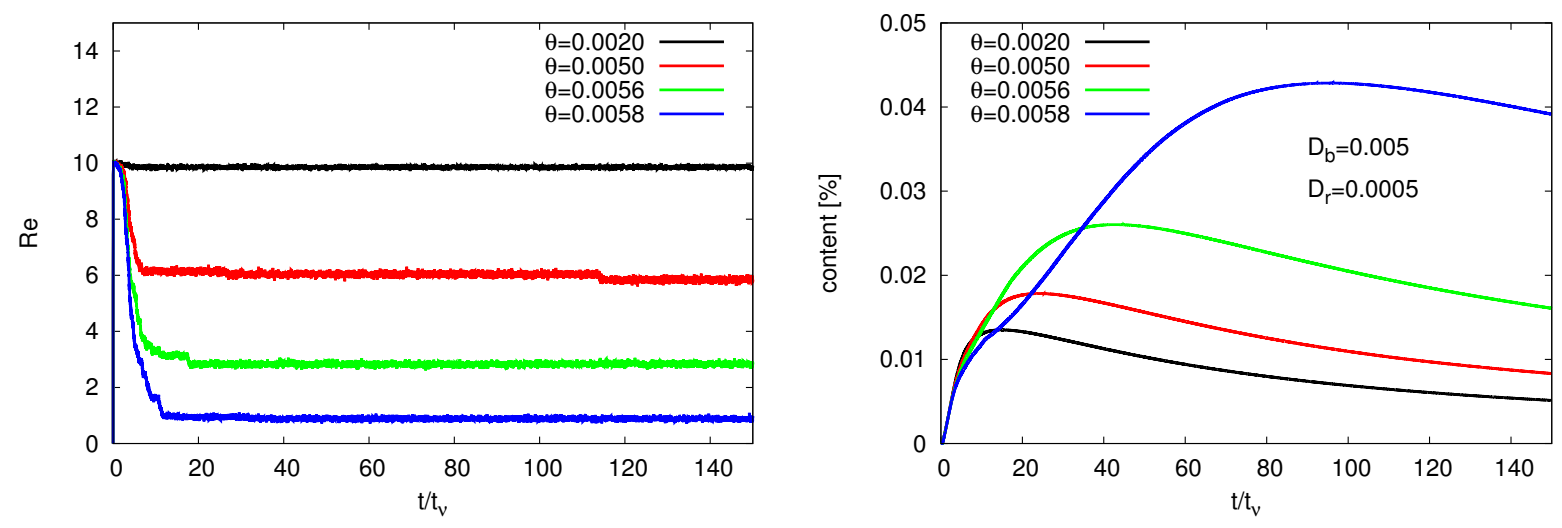

FIG. 7: Direct discharge. Left: time-dependent Reynolds number (absolute value) for different fines concentration $\theta$. Right: cumulative output content.

According to the time scale shown Fig.7, the temporal window explored is in the range of [0-25]s which corresponds to the typical time for an espresso preparation.

\section{Effect of fine concentration $\theta$}

We first select two typical values of diffusion coefficients consistent with the discussion given in Sec.III.A, that is $D_{b}=0.005$ (corresponding to peak/steady Peclet numbers $\left.\mathrm{Pe}_{\text {bulk }}=6000 / 600\right)$ and $D_{r}=0.0005=0.1 D_{b}$, and check the effect of the fines concentration $\theta$ on the the cumulative molecular content. In this section intra-granular diffusion coefficients $D_{s}=0$ so release mechanism is limited to the molecular compounds located inside a grain near the solid/liquid interface. Effect of different intra-granular diffusion coefficients $D_{s}$ will be discussed in the next section.

From Fig.7 (left) it can be seen how for small values of the fines concentration (e.g. $\theta=0.002)$ no transient permeability is observed. The resulting dynamics of the output compound content (Fig.7: right) exhibits a peak value $(\approx 0.014 \%)$ at short times $\left(t^{*} \approx 15\right)$ after which the output compound content in the cup decreases softly.

This peak value is shifted towards larger times and increases in magnitude for increasing $\theta$. For example, for $\theta=0.0058$, peak concentration $(\approx 0.04 \%)$ is reached at dimensionless time $t^{*} \approx 95$. Therefore, the present results shows that incorporation of a finite amount of fines, by inducing a transient permeability of the coffee bed, leads to smaller steady flow rates during filtration $(\mathrm{Re} \approx 1)$. This, in turn, increases the residence time of the flow in contact 


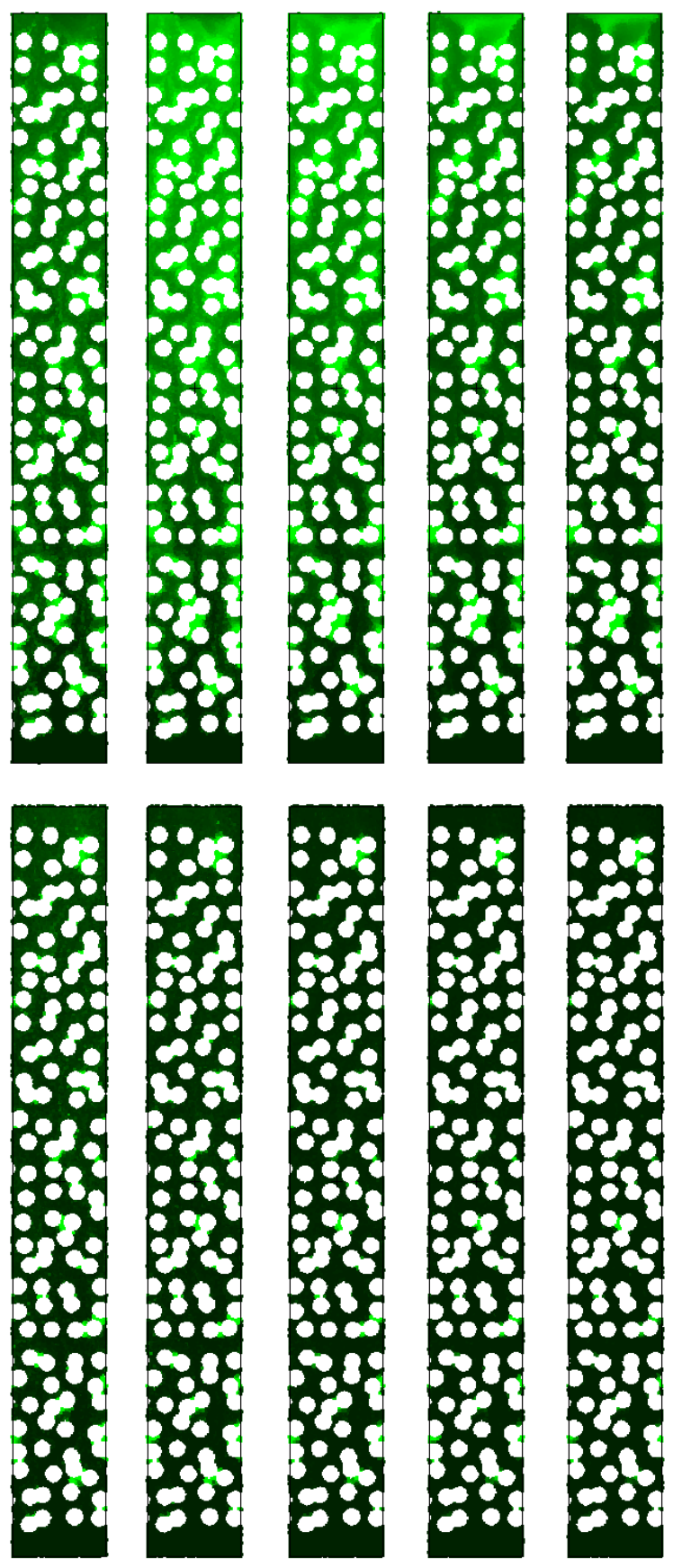

FIG. 8: Effect of $\theta$ on the evolution of the molecular concentration field (green/black: large/zero values). bulk diffusion coefficient $D_{b}=0.005$ and release rate $D_{r}=0.0005$. Top figures: dynamics concentration fields for bulk molecular diffusion coefficient $\theta=0.0058$; bottom figures $\theta=0.002$.

Time frames (left to right) correspond to $t^{*}=12,30,60,90,120$.

with the solid surface of grains, therefore maximizing the molecular release process. In the case of fast flow ( $\mathrm{Re} \approx 10$ for $\theta=0.002)$, water is washed quickly through the coffee bed 

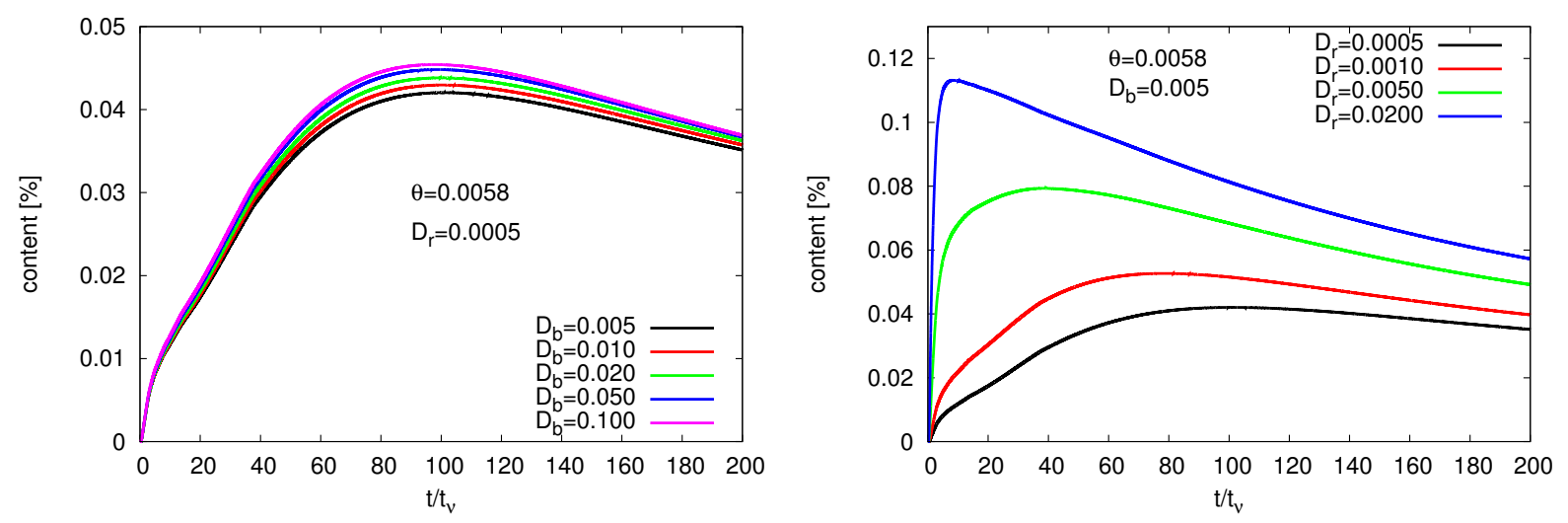

FIG. 9: Direct discharge. Left: cumulative output caffeine content for fixed solid grain's release rate $D_{r}=0.0005$ and $\theta=0.0058$. Right: cumulative output caffeine content for fixed bulk diffusion $D_{r}=0.0005$ and $\theta=0.0058$.

preventing a proper release of substances into the fluid.

Fig.8 shows time frames of the molecular concentration fields (green/black - large/zero values; maximal threshold 0.01) for two different concentrations of fines $\theta=0.0058$ (top) and $\theta=0.002$ (bottom), where other parameters are kept constant. Whereas the molecular compounds is properly released from the solid phase and advected in the case of $\theta=0.0058$, there is no time to be efficiently released in the fast flow condition corresponding to $\theta=$ 0.002. In fact, for $\theta=0.002$ the flow resistance is smaller and, as a consequence, the characteristic time spent by one Lagrangian element in vicinity of the solid-grain interface (where the interfacial transport of solute occurs) will be shorter, leading to a reduced amount of molecular compound released by stripping into a given fluid element.

\section{Effect of bulk diffusion coefficient $D_{b}$ and release rate $D_{r}$}

In a second stage, we focus on the system with fines concentration $\theta=0.0058$ (blue line in Fig.7 left) which reproduces reasonably well the transient flow rate reported in experiments with coffee filtration ${ }^{8}$, i.e. a maximal-peak/minimal-steady Reynolds numbers $\operatorname{Re} \approx 10-1$. In Fig.9 we look at the effect of several parameters on the resulting output cumulative molecular content. In particular, in Fig.9 (left) the cumulative output content is shown for fixed solid grain's release rate $D_{r}=0.0005$ and different bulk diffusion coefficients 


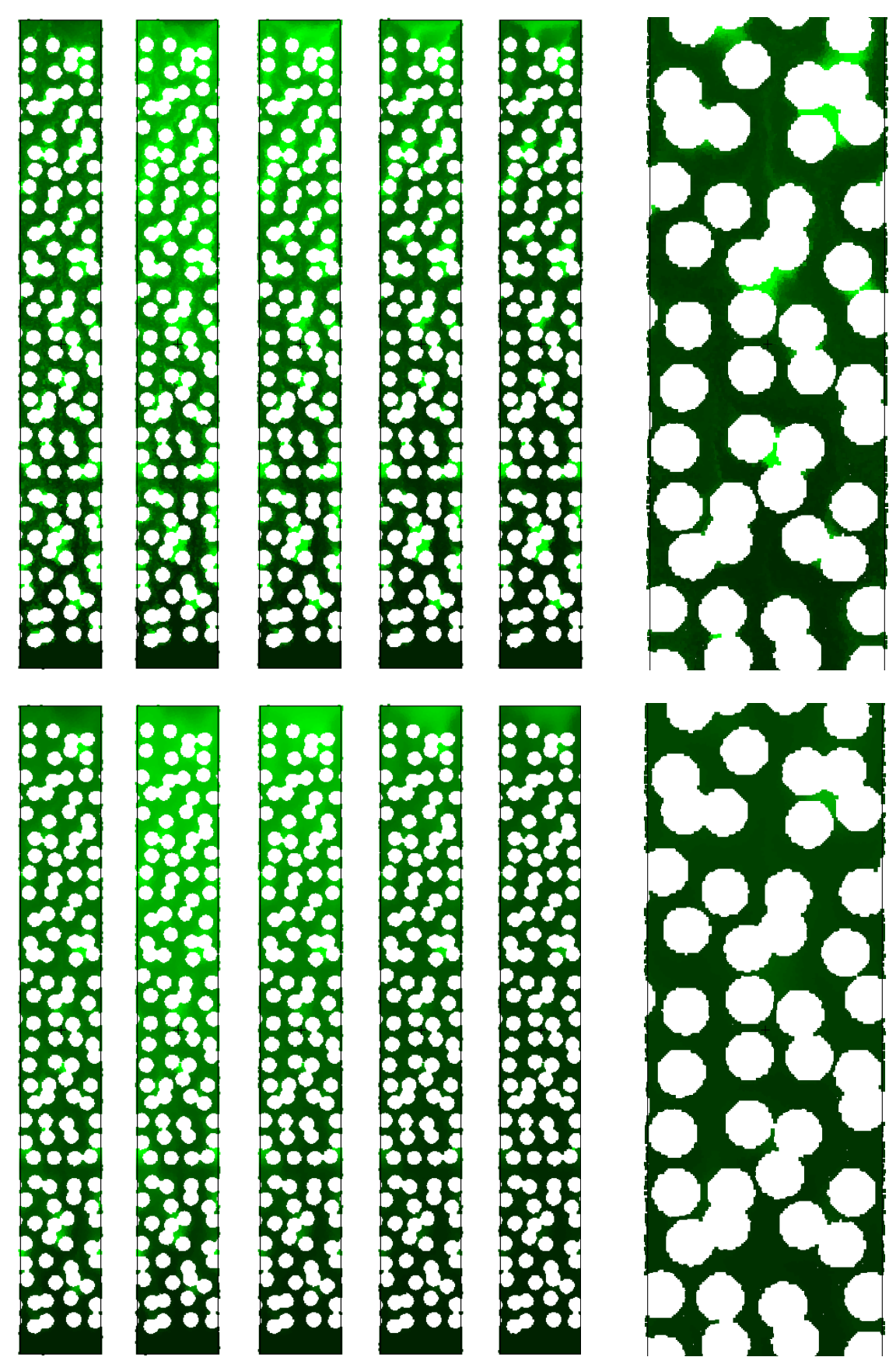

FIG. 10: Effect of $D_{b}$ on the evolution of the molecular concentration field (green/black:

large/zero values). Fine concentration $\theta=0.0058$ and release rate $D_{r}=0.0005$. Top figures: dynamics concentration fields for bulk molecular diffusion coefficient $D_{b}=0.005$; bottom figures $D_{b}=0.01$. Time frames (left to right) correspond to $t^{*}=12,30,60,90,120$. Last frame (right) is an inset at $t^{*}=200$. 
number. It can be seen that $D_{b}$ has only a minor effect on the final output content. There is a small consistent increase (less than $5 \%$ ) in the peak for increasing $D_{b}$ which can be attributed to difference in the release of molecular compounds in areas of stagnating flow. For molecular species released in these areas, the only possible mechanism of escape is by molecular diffusion, i.e. by slowly diffusing into region of large flow where the material is efficiently advected.

Fig.10 shows time frames of the molecular concentration fields for two different bulk diffusion coefficients $D_{b}=0.005$ (top figures) and $D_{b}=0.01$ (bottom figures). In both cases $\theta=0.0058$ and the release rate $D_{r}=0.0005$ are kept constant. By visual inspection, it is clear that bottom concentration frames at large $D_{b}$ are significantly smoother than those at $D_{b}=0.005$ (top). For $D_{b}=0.005$ the diffusive transport mechanism is very slow and material will remain trapped in areas of stagnating flow leading to the smaller values of the overall output content. The last top/bottom plots on the right of Fig.10 represent insets at $t^{*}=120$. As mentioned above, the field at $D_{b}=0.01$ (bottom) is significant smoother; moreover, the regions of residual concentration (present at $D_{b}=0.005$ - top) are almost absent. Note that, being regions of zero flow relatively small (compared to the total liquid domain) this effect is only minor, as suggested by the small increase shown in Fig.9 (left).

More interesting is the effect of the solid/liquid molecular release rate $D_{r}$ on the cumulative output content (Fig.9: right). Here we consider a molecular compound with fixed bulk diffusion $D_{b}=0.005$ at fine concentration $\theta=0.0058$ and look at different release rates coefficients $D_{r} \in[0.0005: 0.02]$. It is clear that $D_{r}$ is the most relevant parameter controlling the final concentration of substance in the cup. For small values of the release coefficient (e.g $D_{r}=0.0005$ : black line) only a small peak $(0.04 \%)$ is reached at relatively long times after which the cumulative content decay very slowly. On the other hand, large values of $D_{r}$ (e.g $D_{r}=0.02$ : blue line) lead to a peak in the order of $0.12 \%$. Moreover, all the material is released very efficiently in the very early stage of filtration $\left(t^{*}<30\right)$ with significant decay occurring later on. Maximization of molecular concentration in the cup can be therefore reached on extremely fast filtration processes by tuning the release mechanism. This is independent on the molecular property of the substance in the fluid (i.e. the diffusion coefficient $D_{b}$ in water). However, it depends strongly on $D_{r}$ and can also depend on the intra-granular diffusion coefficient $D_{s}$ of the molecular compound which indirectly affects 


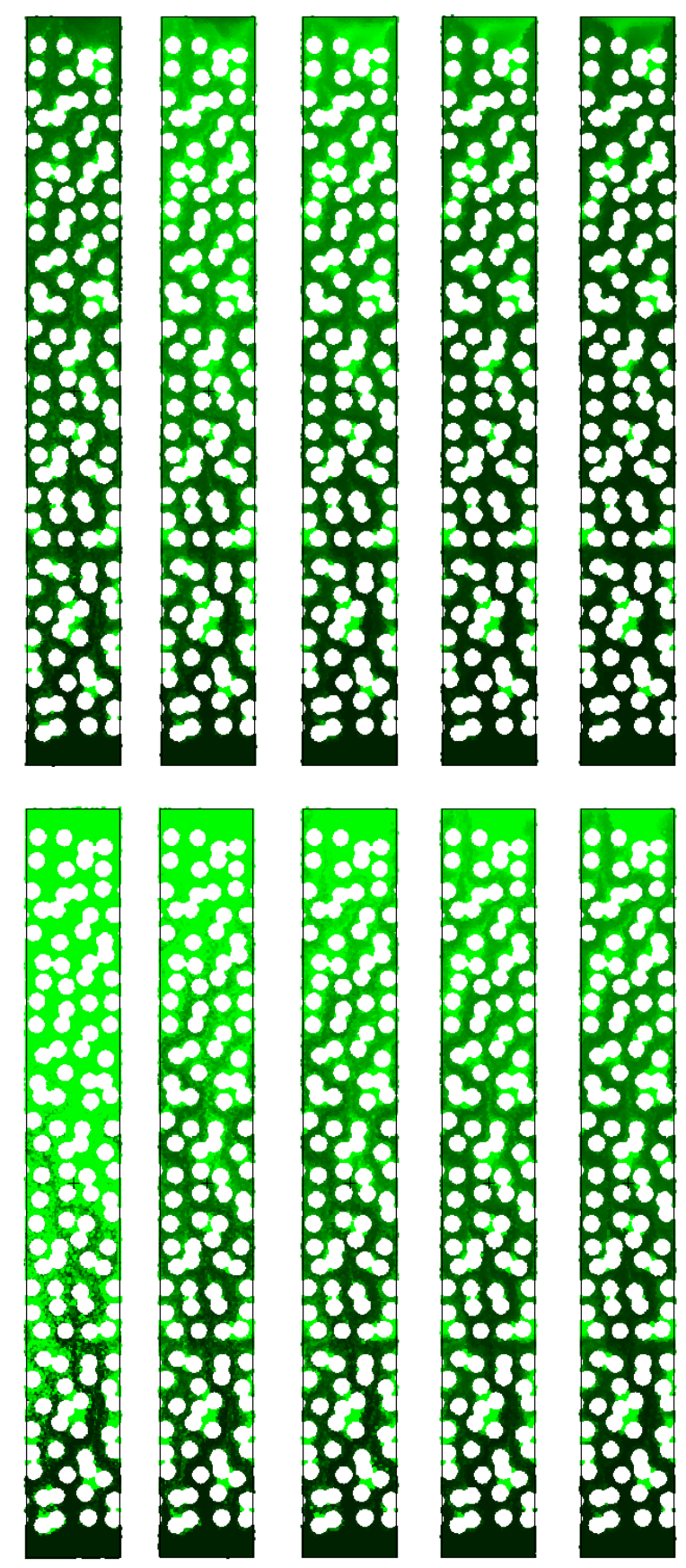

FIG. 11: Effect of $D_{r}$ on the evolution of the molecular concentration field (green/black: large/zero values). Bulk diffusion coefficient $D_{b}=0.005$ and fine concentration $\theta=0.00058$. Top figures: dynamics concentration fields for release rate coefficient $D_{r}=0.0005$; bottom figures $D_{r}=0.02$. Time frames (left to right) correspond to $t^{*}=12,30,60,90,120$. $D_{r}$ (see next Sec.).

Fig.11 shows time frames of the molecular concentration fields for two different release 
rate coefficients $D_{r}=0.0005$ (top figures) and $D_{r}=0.02$ (bottom figures). In both cases $\theta=0.0058$ and the bulk diffusion coefficient $D_{b}=0.005$ are kept constant. As expected, under same hydrodynamic conditions the case $D_{r}=0.02$ is significantly more effective in releasing molecular compounds into the flowing liquid.

\section{Effect of intra-granular diffusion $D_{s}$}

In this section, we look at the effect of a finite intra-granular diffusion coefficient $D_{s}$ on the microstructural dynamics and evolution of the concentration field for a given molecular compound. Fig.12 shows two typical evolutions of the molecular concentration field inside the grains. Violet color corresponds to maximum initial concentration (1.0) whereas red color corresponds to the lowest concentration (0). Top figures correspond to $D_{s}=0.02$; bottom figures: $D_{s}=0$ (no intra-granular diffusion). In all cases, the remaining physical parameters are $D_{b}=0.005, \theta=0.0058$ and $D_{r}=0.02$. These values are chosen in a range delivering realistic dimensionless averaged Reynolds numbers (see Fig. 7) and Schmidt numbers for caffeine as discussed in Sec. III A.

From the bottom figures it can be evinced that only a portion of the internal molecular content is effectively released from the grain. This corresponds to intra-granular regions located near the grain's surfaces, where the washing release mechanism (governed by $D_{r}$ ) is effective already in the very early stage. At longer times the configuration does not change significantly, indicating that the solid/liquid release process has saturated. Given that $D_{s}=0$, there is no diffusion/osmotic mechanism capable of transporting the internal content towards the grain surface where it can be effectively dragged away by the flow and therefore molecular substances which can be described by this low intra-granular diffusion are likely to be extracted in a minor amount during coffee filtration and in the very early stage of the process. This corresponds, for example, to oils which are hydrophobic and remain typically trapped in big amounts inside the grains being only a small fraction present in the beverage ${ }^{7}$.

On the other hand, when intra-granular diffusion is switched-on $D_{s}=0.02$ (top figures), the internal material can diffuse efficiently towards the surface where washing into the liquid takes place. From the figures it is possible to evince also that intra-granular molecular content in this case gradually reduces to zero. Moreover, as it can be seen in the last two 

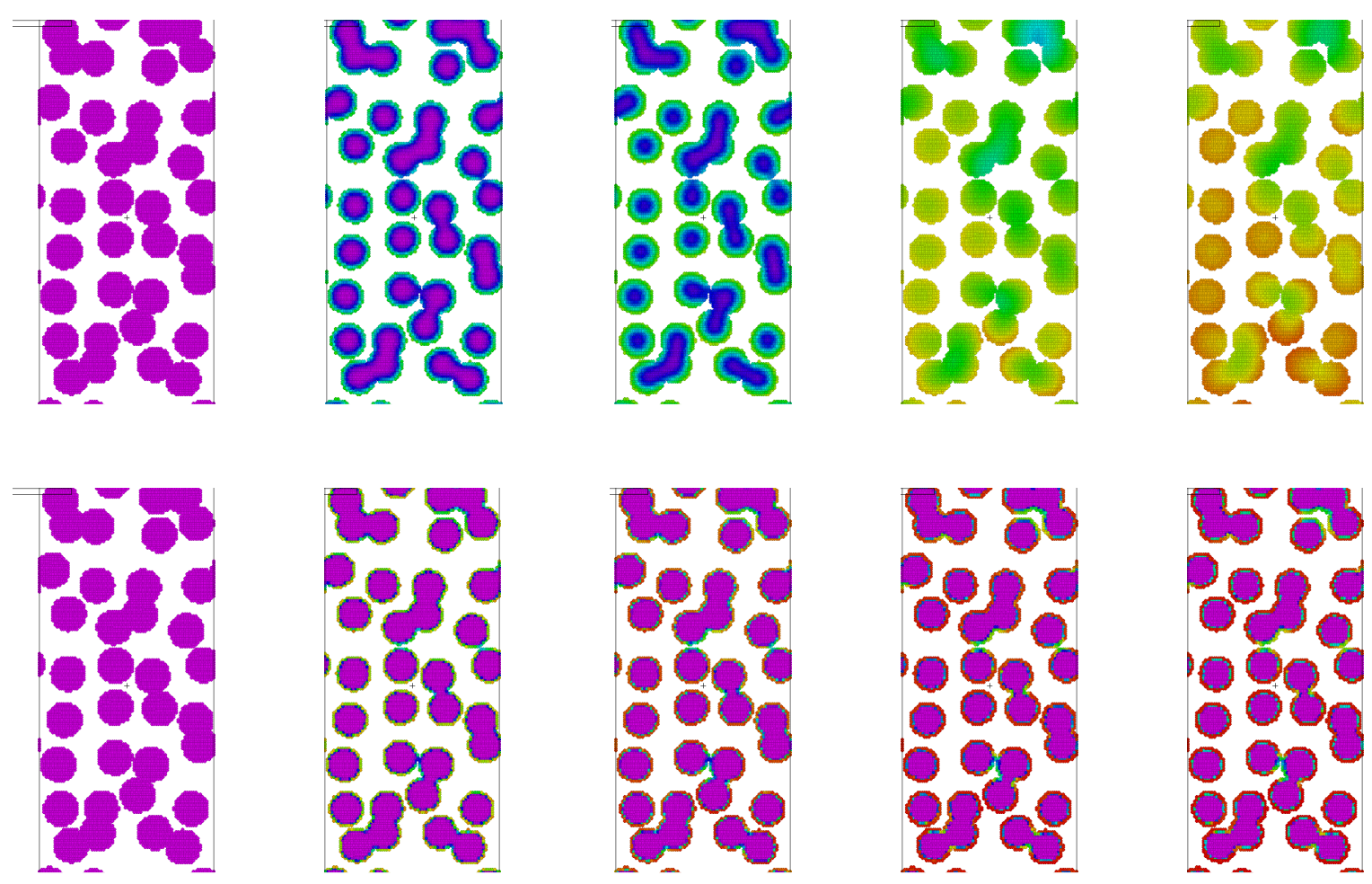

FIG. 12: Effect of the intra-granular diffusion coefficient $D_{s}$ on the evolution of the molecular concentration field (violet/red: large/zero values) inside a solid grain (zoomed areas). Bulk diffusion coefficient in the liquid $D_{b}=0.005$, fine concentration $\theta=0.0058$ and release rate $D_{r}=0.02$ were kept fixed. Top figures: dynamics intra-granular concentration fields for $D_{s}=0.02$; bottom figures $D_{s}=0$. Time frames (left to right) correspond to $t^{*}=0,3,6,30,50$.

As in the previous cases, this direct discharge flow is direct upwards.

top figures on the right, at longer times the molecular removal occurs first in the bottom part of the domain and then gradually invades the upper part. This suggests that grains located near the top filter are more likely to have some residual molecular contents respect to those located far form it. By waiting long enough, all the internal substance will be eventually able to escape the grain's internal core by diffusion and washed out into the liquid. Substances described by this dynamics are typically low molecular weight hydro-soluble and are likely to have nearly $100 \%$ extraction efficiency, as for example the case of caffeine ${ }^{58}$. 
Finally, a comparison of the simulation results with experimental data of three different molecular compounds concentration in the cup provided by Illy $^{63}$ is shown in Fig.13. In particular caffeine, trigonelline and chlorogenic acid are considered. Here we compare the transient extraction efficiency defined as the ratio between the extracted amount of a given molecular compound over its total initial content in the dry grains. As it can be seen in Fig.13, all molecular compounds have $100 \%$ extraction efficiency, however they differ in the relaxation times. For example, trigonelline in our model is well captured by fitting $D_{s}=0.02$ whereas for caffeine an intra-granular diffusion coefficient $D_{s}=0.005$ is more appropriate to match the transient experimental data. By decreasing $D_{s}$ the relaxation times become larger and other compounds dynamics (i.e. chlorogenic acid) can be matched accurately. The case $D_{s}=0$ is also shown as reference. In this case, the extraction converges rapidly to a value approximately equal to $30 \%$ corresponding to all material released from the grain's surfaces only (Fig.12: bottom). This would be the case, for example, of oils or lipids which are hydrophobic. By tuning the effective thickness of the surface layer where the washing release mechanism is active, it is possible to obtain several steady values between 0-100\%.

From Fig.13 it is interesting to note that different compounds are clearly characterized by distinct kinetics which suggests that, depending on molecular specificity, the balance between different compounds is altered if extraction is stopped at different times. Because different compounds are tightly connected to specific flavors, and being taste perception a highly non-linear process ${ }^{64}$, only minor changes in this delicate balance can lead to very different sensorial experience. For example caffeine and trigonelline are typically associated to degree of bitterness, whereas chlorogenic acids to acidic taste ${ }^{64}$. As example, Table II shows the change in proportion among these three compounds, indicating a clear trend for increasing time of espresso preparation. In particular percentage of caffeine content is increasing for increasing preparation time. Percentage of trigonelline has an opposite decreasing trend whereas the chlorogenic acid proportion remains approximately constant. These results indicate clearly that different preparation time for espresso can potentially lead to significant changes in taste perception and therefore the current framework, by quantitatively determining their mass contents, can pave the way for a better flavor-engineering of espresso under unexplored flow processing conditions.

Before to conclude, a few words on the limitation and possible extension of the current modelling framework are in order. The present simple two-dimensional model inevitably 


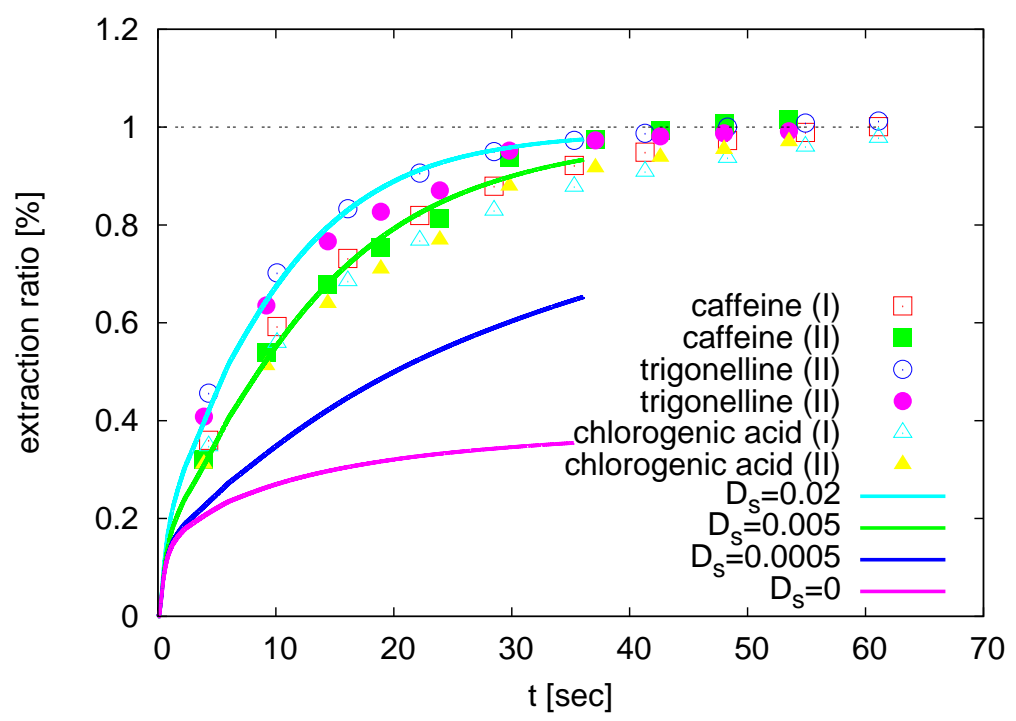

FIG. 13: Direct discharge. Transient extraction ratio of caffeine, trigonelline and chlorogenic acid in the cup. Simulation vs experimental data. SI time units have been obtained by estimating a typical viscous time in the coffee bed $\tau_{\nu}=0.16 \mathrm{~s}$ as previously discussed. All molecular compounds are hydro-soluble and are entirely extracted. Different dynamics are visible indicating that different compounds are released at different times during filtration. The case $D_{s}=0$ in simulation reproduces the scenario of hydrophobic non-soluble substance (e.g. oils) which can be in principle remained trapped into the solid phase.

TABLE II: Proportions of molecular compounds in the cup

\begin{tabular}{|c|c|c|c|}
\hline \hline time [sec] & Caffeine[\%] & Trigonelline [\%] & Chlorogenic acid [\%] \\
\hline 5 & 38.0 & 25.7 & 36.3 \\
10 & 39.5 & 24.6 & 35.9 \\
20 & 40.3 & 23.5 & 36.2 \\
30 & 40.8 & 22.7 & 36.5 \\
\hline \hline
\end{tabular}

lead to qualitative results which can however match well experiments by tuning essentially two model parameters: intra-granular/release and fine concentration. In principle, different porous configurations can lead to different permeabilities. It is clear that the present 2D 
model does not have the ambition of reproducing realistic values of a full 3D coffee bed. Instead, what we chose here is a 'reverse-engineering' approach, where parameters in a simplified (and less computationally expensive) 2D model are tuned to 'reproduce' the relevant physical features (e.g. Reynolds, Schmidt numbers) of a complex 3D system.

In terms of algorithmic complexity for the present model, similar to other particle-based methods with short-range pairwise interactions, it can be reduced down to $\sim \mathcal{O}\left(N N_{\text {neigh }}\right)$ where $N$ is the total number of SPH particles and $N_{\text {neigh }}$ the average number of neighbours if linked-list-cell routines for adaptive neighbours searching are used ${ }^{65}$. A typical simulation on a single processor Intel Xeon E5-2640 2.5GHz takes about 1 day.

In order to move towards more realistic test-cases in the future, an exact prescription of the three-dimensional coffee bed porosity should be obtained by separate experimental technique accessing accurately the internal microstructure. One of these techniques, for example, is X-ray micro-tomography ${ }^{49,50}$, which can provide segmented data for the solid phase to be directly imported into the SPH model. Large-scale simulations of realistic 3D porous configurations can be done as in $^{31}$, by using highly efficient parallel particle-mesh libraries (PPM) for particle-based techniques ${ }^{66}$. This is the subject of a current work and will be presented in the future.

Additionally, the results presented in this paper are limited to isothermal conditions and neglect the non-Newtonian effects of the flowing liquid. Regarding the first point, thermal effects can be incorporated by resorting to an additional internal energy variable per particle as described in the full thermodynamic-consistent model presented in ${ }^{35}$ and link it to the local viscosity in the momentum equation. In connection to the non-Newtonian properties of the liquid, although these are not expected to play a relevant role in the final coffee beverage, they could in principle be modelled in the same way as done recently for shear-thickening fluids ${ }^{46}$ where the liquid's viscosity coefficient was allowed to depend on the local velocity gradient or, as in the case presented in this work, possibly on the local solid fraction of fines. Finally, in the present particle-simulation framework, complex re-modelling of the porous solid phase (e.g. via swelling/erosion) could be directly taken into account by deposition of new fluid particles into the solid phase or by removing existing boundary particles as in the case of erosion. In the latter situation, for example, if local mass concentration associated to boundary particles in the solid phase becomes smaller than a prescribed value, particle identity could be changed (boundary $\rightarrow$ fluid) and allowed to be transported downstream by 
the actual flow. All these physical feature need to be accurately taken into account for a predictive analysis of the complex mesoscopic processes occurring in an espresso extraction and will be the subjects of future investigations.

\section{CONCLUSIONS}

In this work we have presented a mesoscopic particle-based model for the simulation of espresso extraction. In particular, the model incorporates some of the essential physical features of the process, i.e. (i) bimodal granulometry (fines-coarses); (ii) double porosity model of molecular concentrations dynamics (enabling liquid-bulk and intra-granular solid diffusion); (iii) stripping mechanism of solid/liquid molecular release; and (iv) a model of mechanical filter. The correct transient permeability of the coffee bed has been reproduced under direct-inverse discharge conditions, showing the importance of fines migration on the hydrodynamic properties of the percolation and extraction process. Concentration dynamics (i.e. cumulative output content) for different molecular compounds have been explored qualitatively and quantitatively. It was shown that the presence of the fines, leading to larger flow resistance can increase dramatically the residence time of water near the grain surface, therefore maximizing the release process. No visible effect on the cumulative output content dynamics was however detected for different molecular species (i.e. different bulk diffusion coefficients in water). The main parameters governing the molecular dynamics are associated to the solid/liquid stripping release rates as well as intra-granular diffusion.

As an application of the presented modelling framework, the release kinetic of three specific coffee compounds have been simulated and compared to experimental data for a typical and traditional espresso extraction. It was found that the model can reproduce the experimental kinetics by specific choice of the model parameters (e.g. release as well as intra-granular diffusion coefficients). These parameters can be fixed separately for each substance in one extraction process by matching each compound-specific kinetic.

The long-term goal of this work is to use the current framework to control and engineer coffee flavors by monitoring the balance of specific key-odorant or taste-actives compound in the beverage. Decrypting the coffee flavor is a very challenging problem to measure in experimental setup requiring the use of sophisticated methods for sensory-directed chemi- 
cal analysis, including higher sensitivity and selectivity of detection devices, mainly mass spectrometry $(\mathrm{MS})^{64}$. Once key molecular components are experimentally individuated, the present model could be used to explore their change in balance as effect of different applied flows, also in relation to mean sensory response ${ }^{64}$, therefore possibly moving towards a simulated virtual analysis of coffee flavor under different extraction conditions.

\section{ACKNOWLEDGMENTS}

This research is supported by the Basque Government through the BERC 2018-2021 program and by the Spanish Ministry of Science, Innovation and Universities: BCAM Severo Ochoa accreditation SEV-2017-0718 and under the Grant No. RTI2018-094595-B-I00. Furio Suggi Liverani (illycaffè S.p.A.) is acknowledged for helpful discussion.

\section{REFERENCES}

${ }^{1}$ M. Ferrari, F. Ravera, L. Liggieri, and L. Navarini, "Interfacial studies of coffee-based beverages from flavor perception to biofuels," in Surfactant Science and Technology: Retrospects and Prospects, edited by L. S. Romsted (CRC Press, 2014).

${ }^{2}$ A. Parenti, L. Guerrini, P. Masella, S. Spinelli, L. Calamai, and P. Spugnoli, "Comparison of espresso coffee brewing techniques," Journal of Food Engineering 121, 112 - 117 (2014).

${ }^{3}$ E. Illy, "The complexity of coffee," Sci. Am. 286, 92-98 (2002).

${ }^{4}$ M. Ferrari, L. Navarini, L. Liggieri, F. Ravera, and F. Liverani, "Interfacial properties of coffee-based beverages," Food hydrocolloids. 21, 1374-1378 (2007).

${ }^{5}$ A. Voilley and D. Simatos, "Modeling the solubilization process during coffee brewing," Journal of Food Process Engineering 3, 185-198 (1979), https://onlinelibrary.wiley.com/doi/pdf/10.1111/j.1745-4530.1979.tb00238.x.

${ }^{6} \mathrm{G}$. Baldini, Filtrazione non lineare di un fluido attraverso un mezzo poroso deformabile, Master's thesis, University of Florence (1992).

${ }^{7}$ E. Illy and L. Navarini, "Neglected food bubbles: The espresso coffee foam," Food Biophys. 6, 335-348 (2011).

${ }^{8}$ M. Petracco and F. Suggi Liverani, "Espresso coffee brewing dynamics: Development of mathematical and computational model," in Proceedings. 15th International Scientific 
Colloquium on Coffee, ASIC (1993).

${ }^{9} \mathrm{O}$. Fond, "'effect of water and coffee acidity on extraction. dynamics of coffee bed compaction in espresso type extraction," in Proceedings. 16th International Scientific Colloquium on Coffee, ASIC (1995).

${ }^{10}$ A. Fabbri, C. Cevoli, L. Alessandrini, and S. Romani, "Numerical modeling of heat and mass transfer during coffee roasting process," Journal of Food Engineering 105, 264 - 269 (2011).

${ }^{11}$ D. Bottazzi, S. Farina, M. Milani, and L. Montorsi, "A numerical approach for the analysis of the coffee roasting process," Journal of Food Engineering 112, 243 - 252 (2012).

${ }^{12}$ N. O. Oliveros, J. Hernández, F. Sierra-Espinosa, R. Guardián-Tapia, and R. PliegoSolórzano, "Experimental study of dynamic porosity and its effects on simulation of the coffee beans roasting," Journal of Food Engineering 199, 100 - 112 (2017).

${ }^{13}$ R. Cappuccio, G. Cattaneo, G. Erbacci, and U. Jocher, "A parallel implementation of a cellular automata based model for coffee percolation," Parallel Computing 27, 685 - 717 (2001).

${ }^{14}$ R. Rosa, G. von Atzingen, V. Belandria, A. Oliveira, S. Bostyn, and J. Rabi, "Lattice boltzmann simulation of cafestol and kahweol extraction from green coffee beans in high-pressure system," Journal of Food Engineering 176, 88 - 96 (2016), virtualization of Processes in Food Engineering.

${ }^{15}$ S. Bandini, R. Casati, E. Illy, C. Simone, F. Suggi Liverani, and T. F, "A reaction-diffusion computational model to simulate coffee percolation," in Proceedings. 17th International Scientific Colloquium on Coffee, ASIC (1997).

${ }^{16}$ B. Corrochano, J. Melrose, A. Bentley, P. Fryer, and S. Bakalis, "A new methodology to estimate the steady-state permeability of roast and ground coffee in packed beds," Journal of Food Engineering 150, 106 - 116 (2015).

${ }^{17} \mathrm{~F}$. Talamucci, "Flow through a porous medium with mass removal and diffusion," Nonlinear Differential Equations and Applications NoDEA 5, 427-444 (1998).

${ }^{18} \mathrm{~A}$. Fasano and F. Talamucci, "A comprehensive mathematical model for a multispecies flow through ground coffee," SIAM Journal on Mathematical Analysis 31, 251-273 (2000), https://doi.org/10.1137/S0036141098336698.

${ }^{19} \mathrm{~K}$. Moroney, W. Lee, S. Brien, F. Suijver, and J. Marra, "Asymptotic analysis of the dominant mechanisms in the coffee extraction process," SIAM Journal on Applied Mathematics 
76, 2196-2217 (2016).

${ }^{20}$ K. M. Moroney, W. T. Lee, S. B. Brien, F. Suijver, and J. Marra, "Coffee extraction kinetics in a well mixed system," Journal of Mathematics in Industry 7, 3 (2016).

${ }^{21}$ K. Moroney, W. Lee, S. O. Brien, F. Suijver, and J. Marra, "Modelling of coffee extraction during brewing using multiscale methods: An experimentally validated model," Chemical Engineering Science 137, 216-234 (2015).

${ }^{22}$ M. Kuhn, S. Lang, F. Bezold, M. Minceva, and H. Briesen, "Time-resolved extraction of caffeine and trigonelline from finely-ground espresso coffee with varying particle sizes and tamping pressures," Journal of Food Engineering 206, 37 - 47 (2017).

${ }^{23}$ J. M. Aguilera, "Why food microstructure?” Journal of Food Engineering 67, 3- 11 (2005), iV Iberoamerican Congress of Food Engineering (CIBIA IV).

${ }^{24} \mathrm{P}$. Meakin and A. M. Tartakovsky, "Modeling and simulation of porescale multiphase fluid flow and reactive transport in fractured and porous media," Reviews of Geophysics 47 (2009), 10.1029/2008RG000263, https://agupubs.onlinelibrary.wiley.com/doi/pdf/10.1029/2008RG000263.

${ }^{25}$ M. Liu, P. Meakin, and H. Huang, "Dissipative particle dynamics simulation of pore-scale multiphase fluid flow," Water Resources Research 43 (2007), 10.1029/2006WR004856, https://agupubs.onlinelibrary.wiley.com/doi/pdf/10.1029/2006WR004856.

${ }^{26}$ J. Yang and E. S. Boek, "A comparison study of multi-component lattice boltzmann models for flow in porous media applications," Computers Mathematics with Applications 65, $882-890(2013)$.

${ }^{27}$ U. D. Schiller, T. Krüger, and O. Henrich, "Mesoscopic modelling and simulation of soft matter," Soft Matter 14, 9-26 (2018).

${ }^{28}$ Z. Li, W. Pan, and A. M. Tartakovsky, "Particle-based methods for mesoscopic transport processes," in Handbook of Materials Modeling: Applications: Current and Emerging Materials, edited by W. Andreoni and S. Yip (Springer International Publishing, Cham, 2018) pp. 1-20.

${ }^{29}$ J. J. Monaghan, "Smoothed particle hydrodynamics," Reports on Progress in Physics 68, $1703(2005)$.

${ }^{30}$ M. B. Liu and G. R. Liu, "Smoothed particle hydrodynamics (sph): an overview and recent developments," Archives of Computational Methods in Engineering 17, 25-76 (2010). 
${ }^{31}$ A. Vázquez-Quesada and M. Ellero, "Rheology and microstructure of non-colloidal suspensions under shear studied with smoothed particle hydrodynamics," Journal of NonNewtonian Fluid Mechanics 233, 37-47 (2016).

${ }^{32}$ M. Ellero and R. Tanner, "Sph simulations of transient viscoelastic flows at low reynolds number," Journal of Non-Newtonian Fluid Mechanics 132, 61 - 72 (2005).

${ }^{33} \mathrm{X}$. Hu and N. Adams, "A multi-phase sph method for macroscopic and mesoscopic flows," Journal of Computational Physics 213, 844 - 861 (2006).

${ }^{34}$ M. Grmela and H. C. Öttinger, "Dynamics and thermodynamics of complex fluids. i. development of a general formalism," Phys. Rev. E 56, 6620-6632 (1997).

${ }^{35}$ P. Español and M. Revenga, "Smoothed dissipative particle dynamics," Physical Review E 67, 026705 (2003).

${ }^{36}$ A. Vázquez-Quesada, M. Ellero, and P. Español, "Consistent scaling of thermal fluctuations in smoothed dissipative particle dynamics," The Journal of Chemical Physics 130, 034901 (2009).

${ }^{37}$ M. Ellero, P. Español, and E. G. Flekkøy, "Thermodynamically consistent fluid particle model for viscoelastic flows," Phys. Rev. E 68, 041504 (2003).

${ }^{38}$ A. Vázquez-Quesada, M. Ellero, and P. Español, "Smoothed particle hydrodynamic model for viscoelastic fluids with thermal fluctuations," Phys. Rev. E 79, 056707 (2009).

${ }^{39}$ M. Grmela, "Generic guide to the multiscale dynamics and thermodynamics," Journal of Physics Communications 2, 032001 (2018).

${ }^{40}$ A. M. Tartakovsky and P. Meakin, "Pore scale modeling of immiscible and miscible fluid flows using smoothed particle hydrodynamics," Advances in Water Resources 29, 1464 1478 (2006).

${ }^{41}$ A. M. Tartakovsky, P. Meakin, T. D. Scheibe, and R. M. E. West, "Simulations of reactive transport and precipitation with smoothed particle hydrodynamics," Journal of Computational Physics 222, $654-672$ (2007).

${ }^{42}$ A. M. Tartakovsky, P. Meakin, T. D. Scheibe, and B. D. Wood, "A smoothed particle hydrodynamics model for reactive transport and mineral precipitation in porous and fractured porous media," Water Resources Research 43 (2007).

${ }^{43}$ A. M. Tartakovsky, N. Trask, K. Pan, B. Jones, W. Pan, and J. R. Williams, "Smoothed particle hydrodynamics and its applications for multiphase flow and reactive transport in porous media," Computational Geosciences 20, 807-834 (2016). 
${ }^{44}$ L. Navarini, E. Nobile, F. Pinto, A. Scheri, and F. Suggi-Liverani, "Experimental investigation of steam pressure coffee extraction in a stove-top coffee maker," Applied Thermal Engineering 29, 998 - 1004 (2009).

${ }^{45}$ M. Ellero and N. A. Adams, "Sph simulations of flow around a periodic array of cylinders confined in a channel," International Journal for Numerical Methods in Engineering 86, 1027-1040 (2011).

${ }^{46}$ A. Vázquez-Quesada, N. J. Wagner, and M. Ellero, "Planar channel flow of a discontinuous shear-thickening model fluid: Theory and simulation," Physics of Fluids 29, 103104 (2017).

${ }^{47}$ J. P. Morris, P. J. Fox, and Y. Zhu, "Modeling low reynolds number incompressible flows using sph," Journal of computational physics 136, 214-226 (1997).

${ }^{48}$ X. Bian, S. Litvinov, R. Qian, M. Ellero, and N. A. Adams, "Multiscale modeling of particle in suspension with smoothed dissipative particle dynamics," Physics of Fluids 24, $012002(2012)$.

${ }^{49}$ P. Frisullo, J. Laverse, M. Barnabà, L. Navarini, and M. D. Nobile, "Coffee beans microstructural changes induced by cultivation processing: An x-ray microtomographic investigation," Journal of Food Engineering 109, 175 - 181 (2012).

${ }^{50}$ A. J. Griggs, S. M. Davies, P. M. Abbott, M. Coleman, A. P. Palmer, T. L. Rasmussen, and R. Johnston, "Visualizing tephra deposits and sedimentary processes in the marine environment: The potential of x-ray microtomography," Geochemistry, Geophysics, Geosystems 16, 4329-4343 (2015), https://agupubs.onlinelibrary.wiley.com/doi/pdf/10.1002/2015GC006073.

${ }^{51}$ F. Pendolino, Self-assembly of molecules on nanostructured graphene, Ph.D. thesis, Universidad Autónoma de Madrid (2014).

${ }^{52}$ B. Gholami, A. Comerford, and M. Ellero, "Sph simulations of wbc adhesion to the endothelium: the role of haemodynamics and endothelial binding kinetics," Biomechanics and Modeling in Mechanobiology 14, 1317-1333 (2015).

${ }^{53}$ M. Spiro and Y. Y. Chong, "The kinetics and mechanism of caffeine infusion from coffee: the temperature variation of the hindrance factor," Journal of the Science of Food and Agriculture 74, 416-420 (1997).

${ }^{54}$ M.-L. Mateus, M. Rouvet, J.-C. Gumy, and R. Liardon, "Interactions of water with roasted and ground coffee in the wetting process investigated by a combination of physical determinations," Journal of Agricultural and Food Chemistry 55, 2979-2984 (2007). 
${ }^{55}$ M. R, "Nitrogenous components," in Coffee, edited by C. RJ (London: Elsevier Applied Science Publications, 1985) pp. 115-152.

${ }^{56}$ C. MN, "Chlorogenic acids," in Coffee, edited by C. RJ (London: Elsevier Applied Science Publications, 1985) pp. 153-202.

${ }^{57}$ S. Shiraishi, T. Haraguchi, S. Nakamura, H. Kojima, I. Kawasaki, M. Yoshida, and T. Uchida, "Suppression in bitterness intensity of bitter basic drug by chlorogenic acid," Chemical and Pharmaceutical Bulletin 65, 151-156 (2017).

${ }^{58}$ C. Severini, I. Ricci, M. Marone, A. Derossi, and T. De Pilli, "Changes in the aromatic profile of espresso coffee as a function of the grinding grade and extraction time: A study by the electronic nose system," Journal of Agricultural and Food Chemistry 63, 2321-2327 (2015).

${ }^{59}$ W. Pan, I. V. Pivkin, and G. E. Karniadakis, "Single-particle hydrodynamics in dpd: A new formulation," EPL (Europhysics Letters) 84, 10012 (2008).

${ }^{60}$ W. Pan, D. A. Fedosov, G. E. Karniadakis, and B. Caswell, "Hydrodynamic interactions for single dissipative-particle-dynamics particles and their clusters and filaments," Phys. Rev. E 78, 046706 (2008).

${ }^{61}$ E. W. Price, "Tracer caffeine diffusion in aqueous solutions at $298 \mathrm{k}$. the effect of caffeine self-association," J. Chem. Soc., Faraday Trans. 1 85, 415-419 (1989).

${ }^{62}$ F. Rossi, R. Cucciniello, A. Intiso, A. Proto, O. Motta, and N. Marchettini, "Determination of the trichloroethylene diffusion coefficient in water," AIChE Journal 61, 3511-3515, https://onlinelibrary.wiley.com/doi/pdf/10.1002/aic.14861.

${ }^{63}$ L. Navarini, S. Colomban, V. Lonzarich, D. Rivetti, G. Brollo, and F. Suggi-Liverani, "Hyper espresso coffee extraction: adding physics to chemistry." in Proceedings.22nd International Conference on Coffee Science, ASIC (2008).

${ }^{64}$ F. Mestdagh, A. Glabasnia, and P. Giuliano, "Chapter 15 - the brew-extracting for excellence," in The Craft and Science of Coffee, edited by B. Folmer (Academic Press, 2017) pp. $355-380$.

${ }^{65}$ R. W. Hockney and J. W. Eastwood, Computer Simulation Using Particles (Taylor \& Francis, Inc., Bristol, PA, USA, 1988).

${ }^{66}$ I. Sbalzarini, J. Walther, M. Bergdorf, S. Hieber, E. Kotsalis, and P. Koumoutsakos, "Ppm - a highly efficient parallel particle--mesh library for the simulation of continuum systems," Journal of Computational Physics 215, 566 - 588 (2006). 
